\title{
A New Decision Making Method Using Interval-Valued Intuitionistic Fuzzy Cosine Similarity Measure Based on the Weighted Reduced Intuitionistic Fuzzy Sets
}

\author{
Rajkumar VERMA ${ }^{1, *}$, José M. MERIGÓ ${ }^{1,2}$ \\ ${ }^{1}$ Department of Management Control and Information Systems, University of Chile, \\ Av. Diagonal Paraguay 257, Santiago-8330015, Chile \\ ${ }^{2}$ School of Information, Systems and Modelling, Faculty of Engineering \\ and Information Technology, University of Technology Sydney, Sydney, Australia \\ e-mail: rverma@fen.uchile.cl, jmerigo@fen.uchile.cl
}

Received: March 2019; accepted: February 2020

\begin{abstract}
In this paper, we develop a new flexible method for interval-valued intuitionistic fuzzy decision-making problems with cosine similarity measure. We first introduce the interval-valued intuitionistic fuzzy cosine similarity measure based on the notion of the weighted reduced intuitionistic fuzzy sets. With this cosine similarity measure, we are able to accommodate the attitudinal character of decision-makers in the similarity measuring process. We study some of its essential properties and propose the weighted interval-valued intuitionistic fuzzy cosine similarity measure.

Further, the work uses the idea of GOWA operator to develop the ordered weighted interval-valued intuitionistic fuzzy cosine similarity (OWIVIFCS) measure based on the weighted reduced intuitionistic fuzzy sets. The main advantage of the OWIVIFCS measure is that it provides a parameterized family of cosine similarity measures for interval-valued intuitionistic fuzzy sets and considers different scenarios depending on the attitude of the decision-makers. The measure is demonstrated to satisfy some essential properties, which prepare the ground for applications in different areas. In addition, we define the quasi-ordered weighted interval-valued intuitionistic fuzzy cosine similarity (quasi-OWIVIFCS) measure. It includes a wide range of particular cases such as OWIVIFCS measure, trigonometric-OWIVIFCS measure, exponential-OWIVIFCS measure, radical-OWIVIFCS measure. Finally, the study uses the OWIVIFCS measure to develop a new decision-making method to solve real-world decision problems with interval-valued intuitionistic fuzzy information. A reallife numerical example of contractor selection is also given to demonstrate the effectiveness of the developed approach in solving real-life problems.
\end{abstract}

Key words: interval-valued intuitionistic fuzzy sets, weighted reduced intuitionistic fuzzy sets, cosine similarity measure, ordered weighted average operator, multiple attribute decision-making.

\footnotetext{
${ }^{*}$ Corresponding author.
} 


\section{Introduction}

Atanassov (1986) introduced the notion of intuitionistic fuzzy sets (IFSs) as a generalization of the concept of fuzzy sets proposed by Zadeh (1965) in 1965. An IFS is characterized by two functions expressing the degree of membership and the degree of nonmembership, respectively. Later, Atanassov and Gargov (1989) further extended the IFS to interval-valued intuitionistic fuzzy set (IVIFS) whose membership and non-membership functions take values in terms of interval numbers rather than real numbers. Intervalvalued intuitionistic fuzzy sets provide more flexibility to represent vague information in comparison to IFSs. In the past three decades, IFSs and IVIFSs have been successfully applied in different application areas. The basic concepts and practical application of IFSs and IVIFSs in can be found in Atanassov (1994, 2005), Verma and Sharma (2012, 2013a), Vlochos and Sergiadis (2007), Verma and Sharma (2013b, 2013c), Park et al. (2009), Verma and Sharma (2011), Aggarwal and Khan (2016), De et al. (2001), Xu (2010), Ye (2012), Liu and Peng (2017), Zeng et al. (2016), Zhao and Xu (2016), Zhang et al. (2019).

A similarity measure is an essential tool for determining the degree of similarity between two objects. In 2002, Denfeng and Chuntian (2002) introduced the definition of a similarity measure for IFSs and proposed a measure of similarity between IFSs. Mitchell (2003) presented a modified version of Denfeng and Chuntian's similarity for intervalvalued intuitionistic fuzzy sets. Later, Liang and Shi (2003) developed several similarity measures to distinguish different IFSs and discussed the relationship between these measures. Szmidt and Kacprzyk (2013) defined a similarity measure for IFSs using a distance measure. Hong and Kim (1995), Hung and Yang (2004), Xu (2008) defined independently some intuitionistic fuzzy similarity measures based on different distance measures for IFSs. In 2011, Ye (2011) proposed cosine similarity measure for IFSs as the idea parallel to the concept of fuzzy cosine similarity measure Salton and McGill (1983) and applied it to solve pattern recognition and medical diagnosis related problems. Further, Hung and Wang (2012) pointed out some drawbacks of Ye's cosine similarity measure and defined a modified cosine similarity measure for IFSs. Using the idea of generalized ordered weighted aggregation (GOWA) operator Yager (2004), Zhou et al. (2014), proposed the intuitionistic fuzzy ordered weighted cosine similarity (IFOWCS) measure and made a comparative study among different similarity measures.

In many complex decision-making problems, the preference information provided by the decision-makers is often imprecise or uncertain due to the increasing complexity of the social-economic environment or a lack of data about the problem domain or the expert's lack of expertise to precisely express their preferences over the considered objects. In such cases, it is suitable and convenient to express the decision-maker's preference information in terms of IVIFSs. Therefore, it is necessary to pay attention to the study of the similarity measure for IVIFSs. There is some progress in this direction. Xu (2007) generalized some similarity measures of IFSs to IVIFSs, which are based on distance measures for IVIFSs. Zhang et al. (2011) proposed an efficient method to calculate the degree of similarity between IVIFSs based on the Hausdorff metric. Wei et al. (2011) developed a new method to construct the similarity measure for IVIFSs by using entropy function. In 2012, 
Singh (2012) defined a cosine similarity measure for IVIFSs and applied it to solve pattern recognition problems. Further, Ye (2013) studied a new cosine similarity measure with interval-valued intuitionistic fuzzy information and demonstrated its application in multiple attribute decision-making problems. Recently, Liu et al. (2017) proposed the notion of interval-valued intuitionistic fuzzy ordered weighted cosine similarity measure and developed a method to solve group decision-making problems.

Note that the cosine similarity measures introduced by Singh (2012) and Ye (2013) are used only for the middle points and the boundary points of the intervals, respectively, to measure the degree of similarity between two IVIFSs. Due to this limitation, we cannot accommodate the decision maker's attitude in the measuring process. It shows the inability and rigidness of the measures in solving real-world decision problems. So, we need a flexible cosine similarity measure to accommodate the decision maker's attitude preferences in the measuring process under an interval-valued intuitionistic fuzzy environment.

To do so, we first propose a new cosine similarity measure for IVIFSs based on the weighted reduced intuitionistic fuzzy sets (Ye, 2012). Secondly, using the idea of GOWA operator (Yager, 2004), we develop a generalized cosine similarity measure for IVIFSs. We call it 'ordered weighted interval-valued intuitionistic fuzzy cosine similarity (OWIVIFCS) measure'. This extension provides flexibility/choices at the aggregation stage and gives a parameterized family of cosine similarity measures. Furthermore, the work also develops a more general cosine similarity measure between IVIFSs based on quasi-arithmetic means. The applicability of the proposed approach is studied in decisionmaking problems with interval-valued intuitionistic fuzzy information.

The paper is organized as follows. Section 2 briefly reviews the basic concepts related to fuzzy sets, intuitionistic fuzzy sets, interval-valued intuitionistic fuzzy sets, and OWA operators. Section 3 introduces a cosine similarity measure for IVIFSs with some mathematical properties and special cases. Further, a weighted cosine similarity measure for IVIFSs is also defined. In Section 4 we propose the ordered weighted interval-valued intuitionistic fuzzy cosine similarity (OWIVIFCS) measure between two intuitionistic fuzzy sets. Some properties and different families of the OWIVIFCS measure are also analysed. Futhermore, the quasi-OWIVIFCS measure is presented. Section 5, using the OWIVIFCS measure, develops a multiple criteria decision-making model to solve real-world decision problems with interval-valued intuitionistic fuzzy information and illustrate with a numerical example. Section 6 summarizes the main results and conclusions of the paper.

\section{Preliminaries}

In this section, we present some basic concepts related to fuzzy sets, intuitionistic fuzzy sets, interval-valued fuzzy sets, and OWA operators, which will be needed in the following analysis.

Definition 1 (Fuzzy set, Zadeh, 1965). A fuzzy set $\tilde{A}$ in a finite universe of discourse $X=\left\{x_{1}, x_{2}, \ldots, x_{n}\right\}$ is defined by Zadeh as

$$
\tilde{A}=\left\{\left\langle x, \eta_{\tilde{A}}(x)\right\rangle \mid x \in X\right\}
$$


where $\eta_{\tilde{A}}(x): X \rightarrow[0,1]$ is the membership function of $\tilde{A}$. The number $\eta_{\tilde{A}}(x)$ describes the degree of membership of $x \in X$ to $\tilde{A}$.

A cosine similarity measure is defined as the inner product of two vectors divided by the product of their lengths. This is nothing but the cosine of the angle between the vectors representation of two fuzzy sets.

Definition 2 (Cosine similarity measure for FSs, Salton and McGill, 1983). Let $\tilde{A}$ and $\tilde{B}$ be two fuzzy sets in $X=\left\{x_{1}, x_{2}, \ldots, x_{n}\right\}$ having membership values $\eta_{\tilde{A}}\left(x_{i}\right)$ and $\eta_{\tilde{B}}\left(x_{i}\right)$, $i=1,2, \ldots, n$, respectively. A cosine similarity measure between two fuzzy sets $\tilde{A}$ and $\tilde{B}$ analogous to Bhattacharya's distance (Bhattacharya, 1946) is defined as follows:

$$
C_{F S}(A, B)=\frac{\sum_{i=1}^{n} \eta_{\tilde{A}}\left(x_{i}\right) \eta_{\tilde{B}}\left(x_{i}\right)}{\sqrt{\sum_{i=1}^{n} \eta_{\tilde{A}}^{2}\left(x_{i}\right)} \sqrt{\sum_{i=1}^{n} \eta_{\tilde{B}}^{2}\left(x_{i}\right)}} .
$$

Atanassov (1986) introduced the following generalization of fuzzy sets.

Definition 3 (Intuitionistic fuzzy set, Atanassov, 1986). An intuitionistic fuzzy set $A^{*}$ in a finite universe of discourse $X=\left\{x_{1}, x_{2}, \ldots, x_{n}\right\}$ is given by

$$
A^{*}=\left\{\left\langle x, \eta_{A^{*}}(x), \psi_{A^{*}}(x)\right\rangle \mid x \in X\right\},
$$

where $\eta_{A^{*}}: X \rightarrow[0,1]$ and $\psi_{A^{*}}: X \rightarrow[0,1]$ with the condition $0 \leqslant \eta_{A^{*}}(x)+$ $\psi_{A^{*}}(x) \leqslant 1$. For each $x \in X$, the numbers $\eta_{A^{*}}(x)$ and $\psi_{A^{*}}(x)$ denote the degree of membership and degree of non-membership of $x$ to $A^{*}$, respectively. Further, we call $\xi_{A^{*}}(x)=$ $1-\eta_{A^{*}}(x)-\psi_{A^{*}}(x)$, the degree of hesitance or the intuitionistic index of $x \in X$ to $A^{*}$.

For convenience, we abbreviate the set of all IFSs defined in $X$ by $\operatorname{IFS}(X)$.

In 2011, Ye (2013) extended the idea of cosine similarity measure from fuzzy sets to intuitionistic fuzzy set theory and proposed a cosine similarity measure for IFSs. Later, Hung and Wang (2012) pointed out some drawbacks of Ye's cosine similarity measure and defined a modified cosine similarity measure for IFSs as follows:

Definition 4 (Cosine similarity measure for IFSs, Hung and Wang, 2012). Let $A^{*}$ and $B^{*}$ be two intuitionistic fuzzy sets in $X=\left\{x_{1}, x_{2}, \ldots, x_{n}\right\}$ having membership values $\eta_{A^{*}}\left(x_{i}\right)$ and $\eta_{B^{*}}\left(x_{i}\right), i=1,2, \ldots, n$, and non-membership values $\psi_{A^{*}}\left(x_{i}\right)$ and $\psi_{B^{*}}\left(x_{i}\right)$, $i=1,2, \ldots, n$, respectively.

A cosine similarity measure between two intuitionistic fuzzy sets $A^{*}$ and $B^{*}$ is defined as follows:

$$
\begin{aligned}
& C_{I F S}\left(A^{*}, B^{*}\right) \\
& =\frac{1}{n} \sum_{i=1}^{n}\left[\frac{\eta_{A^{*}}\left(x_{i}\right) \eta_{B^{*}}\left(x_{i}\right)+\psi_{A^{*}}\left(x_{i}\right) \psi_{B^{*}}\left(x_{i}\right)+\xi_{A^{*}}\left(x_{i}\right) \xi_{B^{*}}\left(x_{i}\right)}{\sqrt{\eta_{A^{*}}^{2}\left(x_{i}\right)+\psi_{A^{*}}^{2}\left(x_{i}\right)+\xi_{A^{*}}^{2}\left(x_{i}\right)} \sqrt{\eta_{B^{*}}^{2}\left(x_{i}\right)+\psi_{B^{*}}^{2}\left(x_{i}\right)+\xi_{B^{*}}^{2}\left(x_{i}\right)}}\right] .
\end{aligned}
$$


Atanassov and Gargov (1989) introduced the notion of the interval-valued intuitionistic fuzzy set by generalizing the idea of IFSs.

Definition 5 (Interval-valued intuitionistic fuzzy set, Atanassov and Gargov, 1989). Let $X=\left\{x_{1}, x_{2}, \ldots, x_{n}\right\}$ be a finite universe of discourse and $D[0,1]$ denote all the closed subintervals of the interval $[0,1]$. An interval-valued intuitionistic fuzzy set $A$ in $X$ is defined as:

$$
A=\left\{\left\langle x,\left[\eta_{A}^{-}(x), \eta_{A}^{+}(x)\right],\left[\psi_{A}^{-}(x), \psi_{A}^{+}(x)\right]\right\rangle \mid x \in X\right\},
$$

where

$$
\left[\eta_{A}^{-}(x), \eta_{A}^{+}(x)\right] \subseteq[0,1] \quad \text { and }\left[\psi_{A}^{-}(x), \psi_{A}^{+}(x)\right] \subseteq[0,1],
$$

with the condition

$$
0 \leqslant \eta_{A}^{+}(x)+\psi_{A}^{+}(x) \leqslant 1 \quad \text { for any } x \in X .
$$

Here the intervals $\left[\eta_{A}^{-}(x), \eta_{A}^{+}(x)\right]$ and $\left[\psi_{A}^{-}(x), \psi_{A}^{+}(x)\right]$, respectively, denote the degrees of membership and non-membership of $x \in X$ to $A$.

For any $x \in X$, we call the interval

$$
\left[\xi_{A}^{-}(x), \xi_{A}^{+}(x)\right]=\left[1-\eta_{A}^{+}(x)-\psi_{A}^{+}(x), 1-\eta_{A}^{-}(x)-\psi_{A}^{-}(x)\right],
$$

the interval-valued intuitionistic fuzzy index (hesitancy degree) of $x \in X$ to $A$. We will represent the set of all IVIFSs defined in $X$ by $\operatorname{IVIFS}(X)$.

Clearly, if $\eta_{A}^{-}(x)=\eta_{A}^{+}(x)=\eta_{A}(x)$ and $\psi_{A}^{-}(x)=\psi_{A}^{+}(x)=\psi_{A}(x)$, then the given IVIFS $A$ is converted to an ordinary IFS.

In the study of IVIFSs, the set-theoretic operations are defined as follows:

Definition 6 (Set-theoretic operations on IVIFSs, Atanassov and Gargov, 1989). Let $A, B \in \operatorname{IVIFS}(X)$ given by

$$
\begin{aligned}
& A=\left\{\left\langle x,\left[\eta_{A}^{-}(x), \eta_{A}^{+}(x)\right],\left[\psi_{A}^{-}(x), \psi_{A}^{+}(x)\right]\right\rangle \mid x \in X\right\}, \\
& B=\left\{\left\langle x,\left[\eta_{B}^{-}(x), \eta_{B}^{+}(x)\right],\left[\psi_{B}^{-}(x), \psi_{B}^{+}(x)\right]\right\rangle \mid x \in X\right\},
\end{aligned}
$$

then some set operations can be defined as follows:

(i) $A \subseteq B$ if and only if $\eta_{A}^{-}(x) \leqslant \eta_{B}^{-}(x), \eta_{A}^{+}(x) \leqslant \eta_{B}^{+}(x)$ and $\psi_{A}^{-}(x) \geqslant \psi_{B}^{-}(x), \psi_{A}^{+}(x) \geqslant$ $\psi_{B}^{+}(x) \forall x \in X$

(ii) $A=B$, if and only if $A \subseteq B$ and $B \subseteq A$;

(iii) $A^{C}=\left\{\left\langle x,\left[\psi_{A}^{-}(x), \psi_{A}^{+}(x)\right],\left[\eta_{A}^{-}(x), \eta_{A}^{+}(x)\right]\right\rangle \mid x \in X\right\}$; 
(iv) $A \cup B=\left\{\left\langle x,\left[\eta_{A}^{-}(x) \vee \eta_{B}^{-}(x), \eta_{A}^{+}(x) \vee \eta_{B}^{+}(x)\right],\left[\psi_{A}^{-}(x) \wedge \psi_{B}^{-}(x), \psi_{A}^{+}(x) \wedge \psi_{B}^{+}(x)\right]\right\rangle \mid\right.$ $x \in X\}$;

(v) $A \cap B=\left\{\left\langle x,\left[\eta_{A}^{-}(x) \wedge \eta_{B}^{-}(x), \eta_{A}^{+}(x) \wedge \eta_{B}^{+}(x)\right],\left[\psi_{A}^{-}(x) \vee \psi_{B}^{-}(x), \psi_{A}^{+}(x) \vee \psi_{B}^{+}(x)\right]\right\rangle \mid\right.$ $x \in X\}$.

where $\vee, \wedge$ stand for max. and min. operators, respectively.

Singh (2012) defined the cosine similarity measure between IVIFSs $A$ and $B$ as follows

$$
\begin{aligned}
& C_{S}(A, B) \\
& \quad=\frac{1}{n} \sum_{i=1}^{n}\left(\frac{\left(\frac{\eta_{A}^{-}\left(x_{i}\right)+\eta_{A}^{+}\left(x_{i}\right)}{2}\right)\left(\frac{\eta_{B}^{-}\left(x_{i}\right)+\eta_{B}^{+}\left(x_{i}\right)}{2}\right)+\left(\frac{\psi_{A}^{-}\left(x_{i}\right)+\psi_{A}^{+}\left(x_{i}\right)}{2}\right)\left(\frac{\psi_{B}^{-}\left(x_{i}\right)+\psi_{B}^{+}\left(x_{i}\right)}{2}\right)}{\sqrt{\left(\frac{\eta_{A}^{-}\left(x_{i}\right)+\eta_{A}^{+}\left(x_{i}\right)}{2}\right)^{2}+\left(\frac{\eta_{B}^{-}\left(x_{i}\right)+\eta_{B}^{+}\left(x_{i}\right)}{2}\right)^{2}} \sqrt{\left(\frac{\psi_{A}^{-}\left(x_{i}\right)+\psi_{A}^{+}\left(x_{i}\right)}{2}\right)^{2}+\left(\frac{\psi_{B}^{-}\left(x_{i}\right)+\psi_{B}^{+}\left(x_{i}\right)}{2}\right)^{2}}}\right) .
\end{aligned}
$$

In 2013, Ye (2013) proposed a new formula of interval-valued intuitionistic fuzzy cosine similarity measure between two IVIFSs $A$ and $B$ given by

$$
\begin{aligned}
& C_{Y e}(A, B) \\
& =\frac{1}{n} \sum_{i=1}^{n}\left(\begin{array}{c}
\frac{\eta_{A}^{-}\left(x_{i}\right) \eta_{B}^{-}\left(x_{i}\right)+\eta_{A}^{+}\left(x_{i}\right) \eta_{B}^{+}\left(x_{i}\right)+\psi_{A}^{-}\left(x_{i}\right) \psi_{B}^{-}\left(x_{i}\right)}{+\psi_{A}^{+}\left(x_{i}\right) \psi_{B}^{+}\left(x_{i}\right)+\xi_{A}^{-}\left(x_{i}\right) \xi_{B}^{-}\left(x_{i}\right)+\xi_{A}^{+}\left(x_{i}\right) \xi_{B}^{+}\left(x_{i}\right)} \\
\frac{\sqrt{\left(\eta_{A}^{-}\left(x_{i}\right)\right)^{2}+\left(\psi_{A}^{-}\left(x_{i}\right)\right)^{2}+\left(\xi_{A}^{-}\left(x_{i}\right)\right)^{2}+\left(\eta_{A}^{+}\left(x_{i}\right)\right)^{2}+\left(\psi_{A}^{+}\left(x_{i}\right)\right)^{2}+\left(\xi_{A}^{+}\left(x_{i}\right)\right)^{2}}}{\sqrt{\left(\eta_{B}^{-}\left(x_{i}\right)\right)^{2}+\left(\psi_{B}^{-}\left(x_{i}\right)\right)^{2}+\left(\xi_{B}^{-}\left(x_{i}\right)\right)^{2}+\left(\eta_{B}^{+}\left(x_{i}\right)\right)^{2}+\left(\psi_{B}^{+}\left(x_{i}\right)\right)^{2}+\left(\xi_{B}^{+}\left(x_{i}\right)\right)^{2}}}
\end{array}\right) .
\end{aligned}
$$

Ye (2012) developed a method for transforming the interval-valued intuitionistic fuzzy sets into the weighted reduced intuitionistic fuzzy sets. The method is briefly outlined below:

Definition 7 (Method for transforming IVIFSs into the weighted reduced IFSs). Let $A$ be an interval-valued intuitionistic fuzzy set defined in $X$. Also, two weight vectors are $U=$ $\left(u_{1}, u_{2}\right)$ and $V=\left(v_{1}, v_{2}\right), u_{1}, u_{2}, v_{1}, v_{2} \in[0,1]$ with $u_{1}+u_{2}=1$, and $v_{1}+v_{2}=1$. Then, the weighted reduced IFS, denoted by $\widehat{A}$, of an IVIFS $A$ with respect to the adjustable weight values of $u_{1}, u_{2}, v_{1}$ and $v_{2}$ is defined as

$$
\widehat{A}=\left\{\left\langle x, u_{1} \eta_{A}^{-}(x)+u_{2} \eta_{A}^{+}(x), v_{1} \psi_{A}^{-}(x)+v_{2} \psi_{A}^{+}(x)\right\rangle \mid x \in X\right\} .
$$

By adjusting the values of $u_{1}, u_{2}, v_{1}$ and $v_{2}$, an IVIFS $A$ can be converted into the weighted reduced IFS as desired by a decision-maker.

Some special situations:

i. If $u_{1}=1, u_{2}=0, v_{1}=0$ and $v_{2}=1$, we get the pessimistic reduced IFS defined by

$$
\widehat{A}^{P}=\left\{\left\langle x, \eta_{A}^{-}(x), \psi_{A}^{+}(x)\right\rangle \mid x \in X\right\} .
$$


ii. If $u_{1}=0, u_{2}=1, v_{1}=1$ and $v_{2}=0$, we get the optimistic reduced IFS defined by

$$
\widehat{A}^{O}=\left\{\left\langle x, \eta_{A}^{+}(x), \psi_{A}^{-}(x)\right\rangle \mid x \in X\right\} .
$$

iii. If $u_{1}=u_{2}=v_{1}=v_{2}=0.5$, we get the neutral reduced IFS defined by

$$
\widehat{A}^{N}=\left\{\left\langle x, \frac{\eta_{A}^{-}(x)+\eta_{A}^{+}(x)}{2}, \frac{\psi_{A}^{-}(x)+\psi_{A}^{+}(x)}{2}\right\rangle \mid x \in X\right\} .
$$

\subsection{From OWA Operator to the Quasi-OWA Operator}

The OWA operator was introduced by Yager (1988) in 1988, and it provides a parameterized family of aggregation operators between the maximum and the minimum. The OWA operator has been widely used in theory and applications (Merigó, 2012; Yager, 2002; Merigó and Yager, 2013; Merigó and Gil-Lafuente, 2011a, 2011b, 2010; Xu and Da, 2002; Chen et al., 2004; Fodor et al., 1995; Xu and Chen, 2008; Zhou et al., 2013; Zeng et al., 2017; Yu et al., 2015; Merigó and Casanovas, 2011; Xu, 2012; Su et al., 2013; Yager, 1996, 2006; Verma and Merigó, 2019). It can be defined as follows.

Definition 8 (OWA operator, Yager, 1988). An OWA operator of dimension $n$ is a mapping $O W A: R^{n} \rightarrow R$ that has an associated weighting vector $w=\left(w_{1}, w_{2}, \ldots w_{n}\right)$ with $w_{j} \in[0,1]$ and $\sum_{j=1}^{n} w_{j}=1$, such that

$$
O W A\left(a_{1}, a_{2}, \ldots, a_{n}\right)=\sum_{j=1}^{n} w_{j} b_{j}
$$

where $b_{j}$ is the $j$ th largest of the $a_{i}$.

The OWA operator is commutative, monotonic, bounded, and idempotent. Especially, if $w=(1,0, \ldots, 0)^{T}$, then OWA is reduced to the max operator; if $w=(0,0, \ldots, 1)^{T}$, then OWA is reduced to the min. operator, and if $w=(1 / n, 1 / n, \ldots, 1 / n)^{T}$, then OWA becomes an arithmetic average (AA) operator.

Furthermore, in 2004, Yager (2004) developed the idea of generalized ordered weighted aggregation (GOWA) operator. The GOWA operator is an aggregation operator, which includes the ordered weighted aggregation (OWA) operator (Yager, 1988), the ordered weighted geometric (OWG) operator (Xu and Da, 2002) and the ordered weighted harmonic averaging (OWHA) operator (Chen et al., 2004) as its particular cases.

Definition 9 (GOWA operator, Yager, 2004). A GOWA operator of dimension $n$ is a mapping $O W A: R^{n} \rightarrow R$ that has an associated weighting vector $w=\left(w_{1}, w_{2}, \ldots w_{n}\right)$ with $w_{j} \in[0,1]$ and $\sum_{j=1}^{n} w_{j}=1$, such that

$$
\operatorname{GOWA}\left(a_{1}, a_{2}, \ldots, a_{n}\right)=\left(\sum_{j=1}^{n} w_{j} b_{j}^{\delta}\right)^{1 / \delta},
$$

where $b_{j}$ is the $j$ th largest of the $a_{i}$, and $\delta$ is a parameter such that $\delta \in(-\infty, \infty)$. 
The quasi-arithmetic means are an important class of parameterized aggregation operators that have been used extensively in different application areas. It includes a wide range of aggregation operators such as arithmetic, quadratic, geometric, harmonic, rootpower, and exponential. Fodor et al. (1995) defined the quasi-ordered weighted averaging (quasi-OWA) operator as follows.

Definition 10 (Quasi-OWA operator, Fodor et al., 1995). A quasi-OWA operator of dimension $n$ is a mapping Quasi-OWA : $R^{n} \rightarrow R$ that has an associated weighting vector $w=\left(w_{1}, w_{2}, \ldots w_{n}\right)$ with $w_{j} \in[0,1]$ and $\sum_{j=1}^{n} w_{j}=1$, and a continuous strictly monotonic function $g(\bullet)$, such that

$$
\text { Quasi-OWA }\left(a_{1}, a_{2}, \ldots, a_{n}\right)=g^{-1}\left(\sum_{j=1}^{n} w_{j} g\left(b_{j}\right)\right)
$$

where $b_{j}$ is the $j$ th largest of the $a_{i}$.

The quasi-OWA operator is monotonic, commutative, bounded, and idempotent. If we consider different functions $g(\bullet)$ in the quasi-OWA operator, then we can obtain a group of particular cases.

In the next section, using the idea of weighted reduced IFS of an IVIFS, we propose a new similarity measure on interval-valued intuitionistic fuzzy sets, called 'interval-valued intuitionistic fuzzy cosine similarity' measure. One of the most significant features of the IVIFCS is that it can accommodate the decision maker's attitudinal character in the measuring process.

\section{Interval-Valued Intuitionistic Fuzzy Cosine Similarity Based on Weighted Reduced Intuitionistic Fuzzy Sets}

We proceed with the following formal definition:

Definition 11 (Interval-valued intuitionistic fuzzy cosine similarity measure). Let $A$ and $B$ be two IVIFSs defined in a finite universe of discourse $X=\left\{x_{1}, x_{2}, \ldots, x_{n}\right\}$ and two weight vectors be $U=\left(u_{1}, u_{2}\right)$ and $V=\left(v_{1}, v_{2}\right), u_{1}, u_{2}, v_{1}, v_{2} \in[0,1]$ with $u_{1}+u_{2}=1$, and $v_{1}+v_{2}=1$. Then, according to Definition 7 , the weighted reduced IFSs of the IVIFSs $A$ and $B$ are given as

$$
\widehat{A}=\left\{\left\langle x_{i}, u_{1} \eta_{A}^{-}\left(x_{i}\right)+u_{2} \eta_{A}^{+}\left(x_{i}\right), v_{1} \psi_{A}^{-}\left(x_{i}\right)+v_{2} \psi_{A}^{+}\left(x_{i}\right)\right\rangle \mid x_{i} \in X\right\}
$$

and

$$
\widehat{B}=\left\{\left\langle x_{i}, u_{1} \eta_{B}^{-}\left(x_{i}\right)+u_{2} \eta_{B}^{+}\left(x_{i}\right), v_{1} \psi_{B}^{-}\left(x_{i}\right)+v_{2} \psi_{B}^{+}\left(x_{i}\right)\right\rangle \mid x \in X\right\}
$$


Let

$$
\begin{array}{ll}
\eta_{\widehat{A}}^{i}=u_{1} \eta_{A}^{-}\left(x_{i}\right)+u_{2} \eta_{A}^{+}(x), & \psi_{\widehat{A}}^{i}=v_{1} \psi_{A}^{-}\left(x_{i}\right)+v_{2} \psi_{A}^{+}\left(x_{i}\right), \\
\eta_{\widehat{B}}^{i}=u_{1} \eta_{B}^{-}\left(x_{i}\right)+u_{2} \eta_{B}^{+}\left(x_{i}\right), & \psi_{\widehat{B}}^{i}=v_{1} \psi_{B}^{-}\left(x_{i}\right)+v_{2} \psi_{B}^{+}\left(x_{i}\right) .
\end{array}
$$

Thus, the weighted reduced IFSs of the IVIFSs $A$ and $B$ can be rewritten as

$$
\widehat{A}=\left\{\left\langle x_{i}, \eta_{\widehat{A}}^{i}, \psi_{\widehat{A}}^{i}\right\rangle \mid x_{i} \in X\right\} \quad \text { and } \quad \widehat{B}=\left\{\left\langle x_{i}, \eta_{\widehat{B}}^{i}, \psi_{\widehat{B}}^{i}\right\rangle \mid x_{i} \in X\right\}
$$

Analogous to the cosine similarity measure for IFSs given in Eq. (4), an intervalvalued intuitionistic fuzzy cosine similarity measure based on the weighted reduced IFSs of IVIFSs $A$ and $B$ can be defined as follows

$$
\begin{aligned}
& C_{I V I F C S}(\widehat{A}, \widehat{B}) \\
& \quad=\frac{1}{n} \sum_{i=1}^{n}\left(\frac{\eta_{\overparen{A}}^{i} \eta_{\overparen{B}}^{i}+\psi_{\widehat{A}}^{i} \psi_{\widehat{B}}^{i}+\left(1-\eta_{\widehat{A}}^{i}-\psi_{\overparen{A}}^{i}\right)\left(1-\eta_{\overparen{B}}^{i}-\psi_{\overparen{B}}^{i}\right)}{\sqrt{\left(\left(\eta_{\overparen{A}}^{i}\right)^{2}+\left(\psi_{\overparen{A}}^{i}\right)^{2}+\left(1-\eta_{\overparen{A}}^{i}-\psi_{\overparen{A}}^{i}\right)^{2}\right)} \sqrt{\left(\left(\eta_{\overparen{B}}^{i}\right)^{2}+\left(\psi_{\overparen{B}}^{i}\right)^{2}+\left(1-\eta_{\overparen{B}}^{i}-\psi_{\overparen{B}}^{i}\right)^{2}\right)}}\right) .
\end{aligned}
$$

The new measure $C_{I V I F C S}(\widehat{A}, \widehat{B})$ satisfies some important properties, which we study in the following theorems.

Theorem 1. For $A, B \in \operatorname{IVIFS}(X)$, we have

(a) $0 \leqslant C_{I V I F C S}(\widehat{A}, \widehat{B}) \leqslant 1$;

(b) $C_{I V I F C S}(\widehat{A}, \widehat{B})=C_{\text {IVIFCS }}(\widehat{B}, \widehat{A})$;

(c) $C_{I V I F C S}(\widehat{A}, \widehat{B})=1$ if and only if $A=B$, i.e. $\eta_{A}^{-}(x)=\eta_{B}^{-}(x), \eta_{A}^{+}\left(x_{i}\right)=\eta_{B}^{+}\left(x_{i}\right)$ and $\psi_{A}^{-}\left(x_{i}\right)=\psi_{B}^{-}\left(x_{i}\right), \psi_{A}^{+}\left(x_{i}\right)=\psi_{A}^{+}\left(x_{i}\right)$.

Proof. (a) It is evident that the property is true according to the cosine value of Eq. (18).

(b) This follows from the symmetry of $C_{I V I F C S}(\widehat{A}, \widehat{B})$.

(c) First, let $A=B$, i.e. $\eta_{A}^{-}(x)=\eta_{B}^{-}(x), \eta_{A}^{+}\left(x_{i}\right)=\eta_{B}^{+}\left(x_{i}\right)$ and $\psi_{A}^{-}\left(x_{i}\right)=\psi_{B}^{-}\left(x_{i}\right)$, $\psi_{A}^{+}\left(x_{i}\right)=\psi_{A}^{+}\left(x_{i}\right)$. Then from Eq. (18), we get

$$
C_{\operatorname{IVIFCS}}(\widehat{A}, \widehat{B})=1
$$

This proves the 'sufficiency' part of the statement. Next, suppose that $C_{I V I F S}(\widehat{A}, \widehat{B})=1$, i.e.

$$
\begin{aligned}
& \sum_{i=1}^{n}\left(\frac{\eta_{\overparen{A}}^{i} \eta_{\overparen{B}}^{i}+\psi_{\overparen{A}}^{i} \psi_{\overparen{B}}^{i}+\left(1-\eta_{\overparen{A}}^{i}-\psi_{\overparen{A}}^{i}\right)\left(1-\eta_{\overparen{B}}^{i}-\psi_{\overparen{B}}^{i}\right)}{\sqrt{\left(\left(\eta_{\overparen{A}}^{i}\right)^{2}+\left(\psi_{\overparen{A}}^{i}\right)^{2}+\left(1-\eta_{\overparen{A}}^{i}-\psi_{\overparen{A}}^{i}\right)^{2}\right)} \sqrt{\left(\left(\eta_{\overparen{B}}^{i}\right)^{2}+\left(\psi_{\overparen{B}}^{i}\right)^{2}+\left(1-\eta_{\overparen{B}}^{i}-\psi_{\overparen{B}}^{i}\right)^{2}\right)}}\right) \\
& =n \text {, }
\end{aligned}
$$


or

$$
\begin{aligned}
& \eta_{\overparen{A}}^{i} \eta_{\widehat{B}}^{i}+\psi_{\overparen{A}}^{i} \psi_{\overparen{B}}^{i}+\left(1-\eta_{\widehat{A}}^{i}-\psi_{\overparen{A}}^{i}\right)\left(1-\eta_{\overparen{B}}^{i}-\psi_{\overparen{B}}^{i}\right) \\
& =\sqrt{\left(\left(\eta_{\widehat{A}}^{i}\right)^{2}+\left(\psi_{\overparen{A}}^{i}\right)^{2}+\left(1-\eta_{\widehat{A}}^{i}-\psi_{\widehat{A}}^{i}\right)^{2}\right)} \sqrt{\left(\left(\eta_{\overparen{B}}^{i}\right)^{2}+\left(\psi_{\overparen{B}}^{i}\right)^{2}+\left(1-\eta_{\overparen{B}}^{i}-\psi_{\overparen{B}}^{i}\right)^{2}\right)}
\end{aligned}
$$

From the well known Cauchy-Schwarz inequality, we know

$$
\begin{aligned}
& \eta_{\widehat{A}}^{i} \eta_{\overparen{B}}^{i}+\psi_{\overparen{A}}^{i} \psi_{\overparen{B}}^{i}+\left(1-\eta_{\widehat{A}}^{i}-\psi_{\overparen{A}}^{i}\right)\left(1-\eta_{\widehat{B}}^{i}-\psi \psi_{\widehat{B}}^{i}\right) \\
& \quad \leqslant \sqrt{\left(\left(\eta_{\overparen{A}}^{i}\right)^{2}+\left(\psi_{\overparen{A}}^{i}\right)^{2}+\left(1-\eta_{\widehat{A}}^{i}-\psi_{\overparen{A}}^{i}\right)^{2}\right)} \sqrt{\left(\left(\eta_{\overparen{B}}^{i}\right)^{2}+\left(\psi_{\widehat{B}}^{i}\right)^{2}+\left(1-\eta_{\overparen{B}}^{i}-\psi_{\overparen{B}}^{i}\right)^{2}\right)},
\end{aligned}
$$

and becomes equality if and only if

$$
\frac{\eta_{\widehat{A}}^{i}}{\eta_{\widehat{B}}^{i}}=\frac{\psi_{\widehat{A}}^{i}}{\psi_{\widehat{B}}^{i}}=\frac{\left(1-\eta_{\widehat{A}}^{i}-\psi_{\overparen{A}}^{i}\right)}{\left(1-\eta_{\widehat{B}}^{i}-\psi \psi_{\widehat{B}}^{i}\right)}=k
$$

From Eq. (21), we have $\eta_{\widehat{A}}^{i}=k \eta_{\widehat{B}}^{i}, \psi_{\widehat{A}}^{i}=k \psi_{\widehat{B}}^{i},\left(1-\eta_{\widehat{A}}^{i}-\psi_{\widehat{A}}^{i}\right)=k\left(1-\eta_{\widehat{B}}^{i}-\psi \psi_{\widehat{B}}^{i}\right)$ for some positive real number $k$. Since

$$
\eta_{\widehat{A}}^{i}(x)+\psi_{\widehat{A}}^{i}(x)+1-\eta_{\widehat{A}}^{i}(x)-\psi_{\widehat{A}}^{i}(x)=k\left(\eta_{\widehat{B}}^{i}(x)+\psi_{\widehat{B}}^{i}(x)+1-\eta_{\widehat{B}}^{i}(x)-\psi_{\widehat{B}}^{i}(x)\right) .
$$

We have $k=1$, i.e. $\widehat{A}=\widehat{B} \Rightarrow A=B$.

This proves the theorem.

For proof of the further properties, we will consider separation of $X$ into two parts $X_{1}$ and $X_{2}$, such that

$$
\begin{aligned}
& X_{1}=\left\{x_{i} \mid x_{i} \in X, A \subseteq B\right\}, \\
& X_{2}=\left\{x_{i} \mid x_{i} \in X, A \supseteq B\right\} .
\end{aligned}
$$

And note that for all $x_{i} \in X_{1}$,

$$
\begin{aligned}
& {\left[\eta_{A}^{-}\left(x_{i}\right), \eta_{A}^{+}\left(x_{i}\right)\right] \leqslant\left[\eta_{B}^{-}\left(x_{i}\right), \eta_{B}^{+}\left(x_{i}\right)\right] \text { and }} \\
& {\left[\psi_{A}^{-}\left(x_{i}\right), \psi_{A}^{+}\left(x_{i}\right)\right] \geqslant\left[\psi_{B}^{-}\left(x_{i}\right), \psi_{B}^{+}\left(x_{i}\right)\right]}
\end{aligned}
$$

i.e.

$$
\left.\begin{array}{l}
\eta_{A}^{-}\left(x_{i}\right) \leqslant \eta_{B}^{-}\left(x_{i}\right), \eta_{A}^{+}\left(x_{i}\right) \leqslant \eta_{B}^{+}\left(x_{i}\right), \\
\psi_{A}^{-}\left(x_{i}\right) \geqslant \psi_{B}^{-}\left(x_{i}\right), \psi_{A}^{+}\left(x_{i}\right) \geqslant \psi_{B}^{+}\left(x_{i}\right)
\end{array}\right\} .
$$


As also $\forall x_{i} \in X_{2}$,

$$
\begin{aligned}
& {\left[\eta_{A}^{-}\left(x_{i}\right), \eta_{A}^{+}\left(x_{i}\right)\right] \geqslant\left[\eta_{B}^{-}\left(x_{i}\right), \eta_{B}^{+}\left(x_{i}\right)\right] \text { and }} \\
& {\left[\psi_{A}^{-}\left(x_{i}\right), \psi_{A}^{+}\left(x_{i}\right)\right] \leqslant\left[\psi_{B}^{-}\left(x_{i}\right), \psi_{B}^{+}\left(x_{i}\right)\right]}
\end{aligned}
$$

i.e.

$$
\left.\begin{array}{l}
\eta_{A}^{-}\left(x_{i}\right) \geqslant \eta_{B}^{-}\left(x_{i}\right), \eta_{A}^{+}\left(x_{i}\right) \geqslant \eta_{B}^{+}\left(x_{i}\right), \\
\psi_{A}^{-}\left(x_{i}\right) \leqslant \psi_{B}^{-}\left(x_{i}\right), \psi_{A}^{+}\left(x_{i}\right) \leqslant \psi_{B}^{+}\left(x_{i}\right)
\end{array}\right\}
$$

For weighted reduced IFSs of IVIFSs $A$ and $B$, the inequalities (25) and (26) become

$$
\forall x_{i} \in X_{1}, \quad \eta_{\widehat{A}}^{i} \leqslant \eta_{\widehat{B}}^{i}, \quad \psi_{\widehat{A}}^{i} \geqslant \psi_{\widehat{B}}^{i}
$$

and

$$
\forall x_{i} \in X_{2}, \quad \eta_{\widehat{A}}^{i} \geqslant \eta_{\widehat{B}}^{i}, \quad \psi_{\widehat{A}}^{i} \leqslant \psi_{\overparen{B}}^{i} .
$$

Theorem 2. For $A, B \in \operatorname{IVIFS}(X)$,

$C_{I V I F C S}(\widehat{A} \cup \widehat{B}, \widehat{A} \cap \widehat{B})=C_{I V I F C S}(\widehat{A}, \widehat{B})$.

Proof. Using Definition 8, we have

$$
\begin{aligned}
& C_{I V I F C S}(\widehat{A} \cup \widehat{B}, \widehat{A} \cap \widehat{B}) \\
& =\frac{1}{n} \sum_{i=1}^{n}\left(\frac{\eta_{\widehat{A} \cup \widehat{B}}^{i} \eta_{\widehat{A} \cap \widehat{B}}^{i}+\psi_{\widehat{A} \cup \mathcal{B}}^{i} \psi_{\widehat{A} \cap \widehat{B}}^{i}+\left(1-\eta_{\widehat{A} \cup \widehat{B}}^{i}-\psi_{\widehat{A} \cup \widehat{B}}^{i}\right)\left(1-\eta_{\widehat{A} \cap \widehat{B}}^{i}-\psi_{\widehat{A} \cap \widehat{B}}^{i}\right)}{\sqrt{\left(\left(\eta_{\widehat{A} \cup \widehat{B}}^{i}\right)^{2}+\left(\psi_{\widehat{A} \cup \widehat{B}}^{i}\right)^{2}+\left(1-\eta_{\widehat{A} \cup \widehat{B}}^{i}-\psi_{\widehat{A} \cup \widehat{B}}^{i}\right)^{2}\right)} \sqrt{\left(\left(\eta_{\widehat{A} \cap \widehat{B}}^{i}\right)^{2}+\left(\psi_{\widehat{A} \cap \widehat{B}}^{i}\right)^{2}+\left(1-\eta_{\widehat{A} \cap \widehat{B}}^{i}-\psi_{\widehat{A} \cap \widehat{B}}^{i}\right)^{2}\right)}}\right) \\
& =\frac{1}{n}\left[\sum_{x_{i} \in X_{1}}\left(\frac{\eta_{\widehat{B}}^{i} \eta_{\widehat{A}}^{i}+\psi_{\widehat{B}}^{i} \psi_{\widehat{A}}^{i}+\left(1-\eta_{\widehat{B}}^{i}-\psi_{\overparen{B}}^{i}\right)\left(1-\eta_{\widehat{A}}^{i}-\psi_{\overparen{A}}^{i}\right)}{\sqrt{\left(\left(\eta_{\overparen{B}}^{i}\right)^{2}+\left(\psi_{\overparen{B}}^{i}\right)^{2}+\left(1-\eta_{\overparen{B}}^{i}-\psi_{\overparen{B}}^{i}\right)^{2}\right)} \sqrt{\left(\left(\eta_{\overparen{A}}^{i}\right)^{2}+\left(\psi_{\overparen{A}}^{i}\right)^{2}+\left(1-\eta_{\overparen{A}}^{i}-\psi_{\overparen{A}}^{i}\right)^{2}\right)}}\right)\right. \\
& \left.+\sum_{x_{i} \in X_{2}}\left(\frac{\eta_{\overparen{A}}^{i} \eta_{\overparen{B}}^{i}+\psi_{\widehat{A}}^{i} \psi_{\overparen{B}}^{i}+\left(1-\eta_{\widehat{A}}^{i}-\psi_{\overparen{A}}^{i}\right)\left(1-\eta_{\overparen{B}}^{i}-\psi_{\overparen{B}}^{i}\right)}{\sqrt{\left(\left(\eta_{\overparen{A}}^{i}\right)^{2}+\left(\psi_{\overparen{A}}^{i}\right)^{2}+\left(1-\eta_{\overparen{A}}^{i}-\psi_{\overparen{A}}^{i}\right)^{2}\right)} \sqrt{\left(\left(\eta_{\overparen{B}}^{i}\right)^{2}+\left(\psi_{\overparen{B}}^{i}\right)^{2}+\left(1-\eta_{\overparen{B}}^{i}-\psi_{\overparen{B}}^{i}\right)^{2}\right)}}\right)\right] \\
& =C_{I V I F C S}(\widehat{A}, \widehat{B}) \text {. }
\end{aligned}
$$

This proves the theorem.

Theorem 3. For $A, B, C \in \operatorname{IVIFS}(X)$,

(i) $C_{I V I F C S}(\widehat{A} \cup \widehat{B}, \widehat{C}) \leqslant C_{I V I F C S}(\widehat{A}, \widehat{C})+C_{I V I F C S}(\widehat{B}, \widehat{C})$,

(ii) $C_{I V I F C S}(\widehat{A} \cap \widehat{B}, \widehat{C}) \leqslant C_{I V I F C S}(\widehat{A}, \widehat{C})+C_{I V I F C S}(\widehat{B}, \widehat{C})$. 
Proof. We prove (i) only, (ii) can be proved analogously.

(i) Let us consider the expression

$$
\begin{aligned}
& C_{I V I F C S}(\widehat{A}, \widehat{C})+C_{I V I F C S}(\widehat{B}, \widehat{C})-C_{I V I F C S}(\widehat{A} \cup \widehat{B}, \widehat{C}) \\
& =\frac{1}{n} \sum_{i=1}^{n}\left(\frac{\eta_{\widehat{A}}^{i} \eta_{\widehat{C}}^{i}+\psi_{\overparen{A}}^{i} \psi_{\widehat{C}}^{i}+\left(1-\eta_{\widehat{A}}^{i}-\psi_{\overparen{A}}^{i}\right)\left(1-\eta_{\widehat{C}}^{i}-\psi_{\overparen{C}}^{i}\right)}{\sqrt{\left(\left(\eta_{\overparen{A}}^{i}\right)^{2}+\left(\psi_{\overparen{A}}^{i}\right)^{2}+\left(1-\eta_{\overparen{A}}^{i}-\psi_{\overparen{A}}^{i}\right)^{2}\right)} \sqrt{\left(\left(\eta_{\overparen{C}}^{i}\right)^{2}+\left(\psi_{\overparen{C}}^{i}\right)^{2}+\left(1-\eta_{\widehat{C}}^{i}-\psi_{\overparen{C}}^{i}\right)^{2}\right)}}\right) \\
& +\frac{1}{n} \sum_{i=1}^{n}\left(\frac{\eta_{\overparen{B}}^{i} \eta_{\overparen{C}}^{i}+\psi_{\overparen{B}}^{i} \psi_{\widehat{C}}^{i}+\left(1-\eta_{\overparen{B}}^{i}-\psi_{\overparen{B}}^{i}\right)\left(1-\eta_{\overparen{C}}^{i}-\psi_{\overparen{C}}^{i}\right)}{\sqrt{\left(\left(\eta_{\overparen{B}}^{i}\right)^{2}+\left(\psi_{\overparen{B}}^{i}\right)^{2}+\left(1-\eta_{\overparen{B}}^{i}-\psi_{\overparen{B}}^{i}\right)^{2}\right)} \sqrt{\left(\left(\eta_{\overparen{C}}^{i}\right)^{2}+\left(\psi_{\overparen{C}}^{i}\right)^{2}+\left(1-\eta_{\overparen{C}}^{i}-\psi_{\overparen{C}}^{i}\right)^{2}\right)}}\right) \\
& -\frac{1}{n} \sum_{i=1}^{n}\left(\frac{\eta_{\widehat{A} \cup \widehat{B}}^{i} \eta_{\widehat{C}}^{i}+\psi_{\widehat{A} \cup \widehat{B}}^{i} \psi_{\widehat{C}}^{i}+\left(1-\eta_{\widehat{A} \cup \widehat{B}}^{i}-\psi_{\widehat{A} \cup \widehat{B}}^{i}\right)\left(1-\eta_{\widehat{C}}^{i}-\psi_{\widehat{C}}^{i}\right)}{\sqrt{\left(\left(\eta_{\widehat{A} \cup \widehat{B}}^{i}\right)^{2}+\left(\psi_{\widehat{A} \cup \widehat{B}}^{i}\right)^{2}+\left(1-\eta_{\widehat{A} \cup \widehat{B}}^{i}-\psi_{\widehat{A} \cup \widehat{B}}^{i}\right)^{2}\right)} \sqrt{\left(\left(\eta_{\overparen{C}}^{i}\right)^{2}+\left(\psi_{\widehat{C}}^{i}\right)^{2}+\left(1-\eta_{\widehat{C}}^{i}-\psi_{\widehat{C}}^{i}\right)^{2}\right)}}\right) \\
& =\frac{1}{n}\left[\sum_{x_{i} \in X_{1}}\left\{\frac{\eta_{\widehat{A}}^{i} \eta_{\overparen{C}}^{i}+\psi_{\overparen{A}}^{i} \psi_{\widehat{C}}^{i}+\left(1-\eta_{\widehat{A}}^{i}-\psi_{\overparen{A}}^{i}\right)\left(1-\eta_{\widehat{C}}^{i}-\psi_{\overparen{C}}^{i}\right)}{\sqrt{\left(\left(\eta_{\overparen{A}}^{i}\right)^{2}+\left(\psi_{\overparen{A}}^{i}\right)^{2}+\left(1-\eta_{\widehat{A}}^{i}-\psi_{\overparen{A}}^{i}\right)^{2}\right)} \sqrt{\left(\left(\eta_{\overparen{C}}^{i}\right)^{2}+\left(\psi_{\overparen{C}}^{i}\right)^{2}+\left(1-\eta_{\overparen{C}}^{i}-\psi_{\overparen{C}}^{i}\right)^{2}\right)}}\right\}\right. \\
& \left.+\sum_{x_{i} \in X_{2}}\left\{\frac{\eta_{\overparen{B}}^{i} \eta_{\widehat{C}}^{i}+\psi_{\overparen{B}}^{i} \psi_{\widehat{C}}^{i}+\left(1-\eta_{\overparen{B}}^{i}-\psi_{\overparen{B}}^{i}\right)\left(1-\eta_{\widehat{C}}^{i}-\psi_{\overparen{C}}^{i}\right)}{\sqrt{\left(\left(\eta_{\overparen{B}}^{i}\right)^{2}+\left(\psi_{\overparen{B}}^{i}\right)^{2}+\left(1-\eta_{\overparen{B}}^{i}-\psi_{\overparen{B}}^{i}\right)^{2}\right)} \sqrt{\left(\left(\eta_{\overparen{C}}^{i}\right)^{2}+\left(\psi_{\overparen{C}}^{i}\right)^{2}+\left(1-\eta_{\overparen{C}}^{i}-\psi_{\overparen{C}}^{i}\right)^{2}\right)}}\right\}\right]
\end{aligned}
$$

$\geqslant 0$.

This proves the theorem.

Theorem 4. For $A, B, C \in \operatorname{IVIFS}(X)$,

$C_{I V I F S}(\widehat{A} \cup \widehat{B}, \widehat{C})+C_{I V I F S}(\widehat{A} \cap \widehat{B}, \widehat{C})=C_{I V I F S}(\widehat{A}, \widehat{C})+C_{I V I F S}(\widehat{B}, \widehat{C})$

Proof. From Definition 8, we have

$C_{I V I F S}(\widehat{A} \cup \widehat{B}, \widehat{C})$

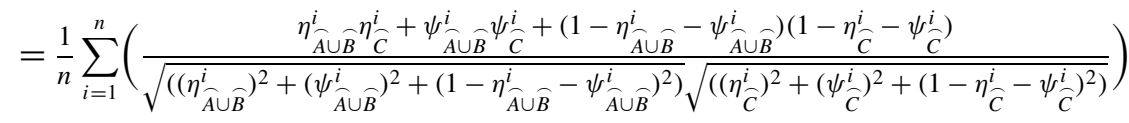

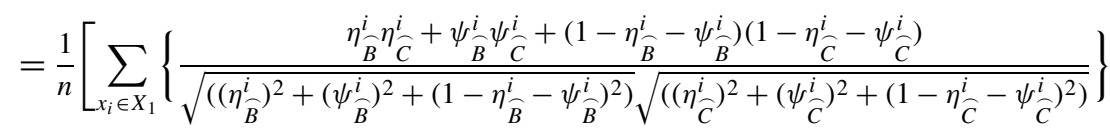

$$
\begin{aligned}
& +\sum_{x_{i} \in X_{2}}\left\{\frac{\eta_{\widehat{A}}^{i} \eta_{\overparen{C}}^{i}+\psi_{\overparen{A}}^{i} \psi_{\widehat{C}}^{i}+\left(1-\eta_{\widehat{A}}^{i}-\psi_{\widehat{A}}^{i}\right)\left(1-\eta_{\widehat{C}}^{i}-\psi_{\overparen{C}}^{i}\right)}{\sqrt{\left(\left(\eta_{\overparen{A}}^{i}\right)^{2}+\left(\psi_{\overparen{A}}^{i}\right)^{2}+\left(1-\eta_{\widehat{A}}^{i}-\psi_{\overparen{A}}^{i}\right)^{2}\right)} \sqrt{\left(\left(\eta_{\overparen{C}}^{i}\right)^{2}+\left(\psi_{\overparen{C}}^{i}\right)^{2}+\left(1-\eta_{\overparen{C}}^{i}-\psi_{\overparen{C}}^{i}\right)^{2}\right)}}\right\},
\end{aligned}
$$


and

$$
\begin{aligned}
& C_{I V I F S}(\widehat{A} \cap \widehat{B}, \widehat{C}) \\
& =\frac{1}{n} \sum_{i=1}^{n}\left(\frac{\eta_{\widehat{A} \cap \widehat{B}}^{i} \eta_{\widehat{C}}^{i}+\psi_{\widehat{A} \cap \widehat{B}}^{i} \psi_{\widehat{C}}^{i}+\left(1-\eta_{\widehat{A} \cap \widehat{B}}^{i}-\psi_{\widehat{A} \cap \widehat{B}}^{i}\right)\left(1-\eta_{\widehat{C}}^{i}-\psi_{\widehat{C}}^{i}\right)}{\sqrt{\left(\left(\eta_{\widehat{A} \cap \widehat{B}}^{i}\right)^{2}+\left(\psi_{\widehat{A} \cap \widehat{B}}^{i}\right)^{2}+\left(1-\eta_{\widehat{A} \cap \widehat{B}}^{i}-\psi_{\widehat{A} \cap \widehat{B}}^{i}\right)^{2}\right)} \sqrt{\left(\left(\eta_{\widehat{C}}^{i}\right)^{2}+\left(\psi_{\widehat{C}}^{i}\right)^{2}+\left(1-\eta_{\widehat{C}}^{i}-\psi_{\widehat{C}}^{i}\right)^{2}\right)}}\right) \\
& =\frac{1}{n}\left[\sum_{x_{i} \in X_{1}}\left\{\frac{\mu_{\overparen{A}}^{i} \mu_{\overparen{C}}^{i}+v_{\widehat{A}}^{i} v_{\overparen{C}}^{i}+\left(1-\mu_{\widehat{A}}^{i}-v_{\overparen{A}}^{i}\right)\left(1-\mu_{\widehat{C}}^{i}-v_{\overparen{C}}^{i}\right)}{\sqrt{\left(\left(\mu_{\overparen{A}}^{i}\right)^{2}+\left(v_{\overparen{A}}^{i}\right)^{2}+\left(1-\mu_{\overparen{A}}^{i}-v_{\overparen{A}}^{i}\right)^{2}\right)} \sqrt{\left(\left(\mu_{\overparen{C}}^{i}\right)^{2}+\left(v_{\overparen{C}}^{i}\right)^{2}+\left(1-\mu_{\overparen{C}}^{i}-v_{\overparen{C}}^{i}\right)^{2}\right)}}\right\}\right. \\
& \left.+\sum_{x_{i} \in X_{2}}\left\{\frac{\mu_{\overparen{B}}^{i} \mu_{\overparen{C}}^{i}+v_{\widehat{B}}^{i} v_{\widehat{C}}^{i}+\left(1-\mu_{\widehat{B}}^{i}-v_{\overparen{B}}^{i}\right)\left(1-\mu_{\overparen{C}}^{i}-v_{\widehat{C}}^{i}\right)}{\sqrt{\left(\left(\mu_{\overparen{B}}^{i}\right)^{2}+\left(v_{\overparen{B}}^{i}\right)^{2}+\left(1-\mu_{\overparen{B}}^{i}-v_{\overparen{B}}^{i}\right)^{2}\right)} \sqrt{\left(\left(\mu_{\overparen{C}}^{i}\right)^{2}+\left(v_{\overparen{C}}^{i}\right)^{2}+\left(1-\mu_{\overparen{C}}^{i}-v_{\overparen{C}}^{i}\right)^{2}\right)}}\right\}\right] .
\end{aligned}
$$

Adding Eq. (30) and Eq. (31), we get the result.

This proves the theorem.

Theorem 5. For $A, B \in \operatorname{IVIFS}(X)$,

(a) $C_{I V I F S}(\widehat{A}, \widehat{B})=C_{I V I F S}\left(\widehat{A}^{C}, \widehat{B}^{C}\right)$;

(b) $C_{I V I F S}\left(\widehat{A}, \widehat{B}^{C}\right)=C_{I V I F S}\left(\widehat{A}^{C}, \widehat{B}\right)$;

(c) $C_{I V I F S}(\widehat{A}, \widehat{B})+C_{I V I F S}\left(\widehat{A}^{C}, \widehat{B}\right)=C_{I V I F S}\left(\widehat{A}^{C}, \widehat{B}^{C}\right)+C_{I V I F S}\left(\widehat{A}, \widehat{B}^{C}\right)$,

where $\widehat{A}^{C}$ and $\widehat{B}^{C}$ represent the complement of the weighted reduced IFSs of IVIFSs $A$ and $B$, respectively.

Proof. (a) It follows from the relation of membership and non-membership of an element in a set and its complement.

(b) It directly follows from Definition 11.

(c) It simply follows (a) and (b).

This proves the theorem.

By adjusting the values of $u_{1}, u_{2}, v_{1}$ and $v_{2}$, we can obtain an interval-valued intuitionistic fuzzy cosine similarity measure between the IVIFSs $A$ and $B$ as desired by a decision-maker.

i. If $u_{1}=1, u_{2}=0, v_{1}=0$ and $v_{2}=1$, then we get the pessimistic interval-valued intuitionistic fuzzy cosine similarity measure given by

$$
\begin{aligned}
& P C_{\operatorname{IVIFCS}}(\widehat{A}, \widehat{B}) \\
& \quad=\frac{1}{n} \sum_{i=1}^{n}\left(\frac{\eta_{A}^{-} \eta_{B}^{-}+\psi_{A}^{+} \psi_{B}^{+}+\left(1-\eta_{A}^{-}-\psi_{A}^{+}\right)\left(1-\eta_{B}^{-}-\psi_{B}^{+}\right)}{\sqrt{\left(\left(\eta_{A}^{-}\right)^{2}+\left(\psi_{A}^{+}\right)^{2}+\left(1-\eta_{A}^{-}-\psi_{A}^{+}\right)^{2}\right)} \sqrt{\left(\left(\eta_{B}^{-}\right)^{2}+\left(\psi_{B}^{+}\right)^{2}+\left(1-\eta_{B}^{-}-\psi_{B}^{+}\right)^{2}\right)}}\right) .
\end{aligned}
$$


ii. If $u_{1}=0, u_{2}=1, v_{1}=1$ and $v_{2}=0$, then we obtain the optimistic interval-valued intuitionistic fuzzy cosine similarity measure given by

$$
\begin{aligned}
& O C_{I V I F C S}(\widehat{A}, \widehat{B}) \\
& \quad=\frac{1}{n} \sum_{i=1}^{n}\left(\frac{\eta_{A}^{+} \eta_{B}^{+}+\psi_{A}^{-} \psi_{B}^{-}+\left(1-\eta_{A}^{+}-\psi_{A}^{-}\right)\left(1-\eta_{B}^{+}-\psi_{B}^{-}\right)}{\sqrt{\left(\left(\eta_{A}^{+}\right)^{2}+\left(\psi_{A}^{-}\right)^{2}+\left(1-\eta_{A}^{+}-\psi_{A}^{-}\right)^{2}\right)} \sqrt{\left(\left(\eta_{B}^{+}\right)^{2}+\left(\psi_{B}^{-}\right)^{2}+\left(1-\eta_{B}^{+}-\psi_{B}^{-}\right)^{2}\right)}}\right) .
\end{aligned}
$$

iii. If $u_{1}=u_{2}=\frac{1}{2}$, and $v_{1}=v_{2}=\frac{1}{2}$, then the neutral interval-valued intuitionistic fuzzy cosine similarity measure is obtained as

$N C_{I V I F C}(\widehat{A}, \widehat{B})$

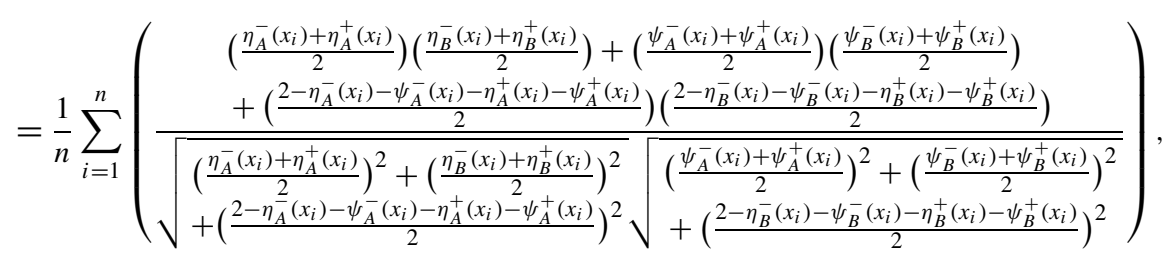

iv. If $\eta_{A}^{-}\left(x_{i}\right)=\eta_{A}^{+}\left(x_{i}\right), \eta_{B}^{-}\left(x_{i}\right)=\eta_{B}^{+}\left(x_{i}\right)$ and $\psi_{A}^{-}\left(x_{i}\right)=\psi_{A}^{+}\left(x_{i}\right), \psi_{B}^{-}\left(x_{i}\right)=\psi_{B}^{+}\left(x_{i}\right)$ $\forall i=1,2, \ldots, n$, then $C_{I V I F C S}(\widehat{A}, \widehat{B})$ reduces to intuitionistic fuzzy cosine similarity measure defined by Ye (2011).

\section{Weighted interval-valued intuitionistic fuzzy cosine similarity measure:}

Assume that the elements in the universe of discourse $X=\left\{x_{1}, x_{2}, \ldots, x_{n}\right\}$ have the weight vector $\omega=\left(\omega_{1}, \omega_{2}, \ldots, \omega_{n}\right)^{T}$ such that $\omega_{i} \geqslant 0$ and $\sum_{i=1}^{n} \omega_{i}=1$. The weighted interval-valued intuitionistic fuzzy cosine similarity (WIVIFCS) measure based on the weighted reduced IFSs of IVIFSs $A$ and $B$ is defined as

$$
\begin{aligned}
& C_{\text {IVIFCS }}^{\omega}(\widehat{A}, \widehat{B}) \\
& \quad=\sum_{i=1}^{n} \omega_{i}\left(\frac{\eta_{\overparen{A}}^{i} \eta_{\widehat{B}}^{i}+\psi_{\overparen{A}}^{i} \psi_{\widehat{B}}^{i}+\left(1-\eta_{\widehat{A}}^{i}-\psi_{\overparen{A}}^{i}\right)\left(1-\eta_{\widehat{B}}^{i}-\psi_{\widehat{B}}^{i}\right)}{\sqrt{\left(\left(\eta_{\overparen{A}}^{i}\right)^{2}+\left(\psi_{\overparen{A}}^{i}\right)^{2}+\left(1-\eta_{\overparen{A}}^{i}-\psi_{\overparen{A}}^{i}\right)^{2}\right)} \sqrt{\left(\left(\eta_{\overparen{B}}^{i}\right)^{2}+\left(\psi_{\overparen{B}}^{i}\right)^{2}+\left(1-\eta_{\overparen{B}}^{i}-\psi_{\overparen{B}}^{i}\right)^{2}\right)}}\right) .
\end{aligned}
$$

Note. (i) If $\omega=(1 / n, 1 / n, \ldots, 1 / n)^{T}$, then the measure defined in Eq. (35) is reduced to measure given in Eq. (18).

Obviously, the $C_{I V I F C S}^{\omega}(\widehat{A}, \widehat{B})$ also satisfies the following properties: 
Theorem 6. For $A, B \in \operatorname{IVIFS}(X)$,

(a) $0 \leqslant C_{I V I F C S}^{\omega}(\widehat{A}, \widehat{B}) \leqslant 1$;

(b) $C_{I V I F C S}^{\omega}(\widehat{A}, \widehat{B})=C_{I V I F C S}^{\omega}(\widehat{B}, \widehat{A})$;

(c) $C_{I V I F C S}^{\omega}(\widehat{A}, \widehat{B})=1$ if $A=B$, i.e. $\eta_{A}^{-}(x)=\eta_{B}^{-}(x), \eta_{A}^{+}(x)=\eta_{B}^{+}(x)$ and $\psi_{A}^{-}(x)=$ $\psi_{B}^{-}(x), \psi_{A}^{+}(x)=\psi_{B}^{+}(x) \forall i=1,2, \ldots, n$.

Proof. These properties can be proved easily on lines similar to the proof of Theorem 1.

Note that the cosine similarity measures defined in Eq. (18) and Eq. (35) are used the arithmetic average (AA) and weighted average (WA) for the normalization process. These measures do not provide flexibility/choices to the user in the aggregation stage. The OWA operator is a parameterized mean-like aggregation operator that reflects the uncertain nature of the decision-maker with the ability to generate an aggregating result lying between two extremes of minimum and maximum. In the past few years, the OWA operator has been used to normalize different measures, including distance measures (Merigó and Yager, 2013; Merigó and Gil-Lafuente, 2011a, 2011b; Xu and Chen, 2008; Zhou et al., 2013; Zeng et al., 2017; Yu et al., 2015; Merigó and Casanovas, 2011; Xu, 2012), similarity measures (Zhou et al., 2014; Liu et al., 2017; Su et al., 2013), adequacy coefficient (Merigó and Gil-Lafuente, 2010), variance (Yager, 1996, 2006; Verma and Merigó, 2019). Motivated by the idea of generalized OWA operator (Yager, 2004), next, we propose an ordered weighted cosine similarity measure between IVIFSs. It is a similarity measure that cannot only emphasize the importance of the ordered position of each similarity value but also provide a parameterized family of cosine similarity between IVIFSs.

\section{Ordered Weighted Interval-Valued Intuitionistic Fuzzy Cosine Similarity (OWIVIFCS)}

Let $A$ and $B$ be two IVIFSs in the finite universe of discourse $X=\left\{x_{1}, x_{2}, \ldots, x_{n}\right\}$ and two weight vectors be $U=\left(u_{1}, u_{2}\right)$ and $V=\left(v_{1}, v_{2}\right), u_{1}, u_{2}, v_{1}, v_{2} \in[0,1]$ with $u_{1}+u_{2}=1$, and $v_{1}+v_{2}=1$. Further, assume that $\widehat{A}=\left\{\left\langle x_{i}, \eta_{\widehat{A}}^{i}, \psi_{\widehat{A}}^{i}\right\rangle \mid x_{i} \in X\right\}$ and $\widehat{B}=\left\{\left\langle x, \eta_{\widehat{B}}^{i}, \psi_{\widehat{B}}^{i}\right\rangle \mid x_{i} \in X\right\}$ denote the weighted reduced IFSs of the IVIFSs $A$ and $B$. Using the idea of generalized OWA (Yager, 2004), we propose with the following formal definition:

Definition 12 (Ordered weighted interval-valued intuitionistic fuzzy cosine similarity measure). A OWIVIFCS measure based on the weighted reduced IFSs of IVIFSs is a mapping OWIVIFCS : IVIFS $(X) \times \operatorname{IVIFS}(X) \rightarrow[0,1]$ that has an associated weighting vector $w=\left(w_{1}, w_{2}, \ldots, w_{n}\right)$ with $w_{j} \in[0,1]$ and $\sum_{j=1}^{n} w_{j}=1$, and defined according to the following formula:

$$
C_{\text {OWIVIFCS }}^{\delta}(\widehat{A}, \widehat{B})=\left[\sum_{j=1}^{n} w_{j}\left(C_{I V I F C S}\left(\widehat{A}_{\sigma(j)}, \widehat{B}_{\sigma(j)}\right)\right)^{\delta}\right]^{1 / \delta}, \quad \delta>0,
$$


where $(\sigma(1), \sigma(2), \ldots, \sigma(n))$ is any permutation of $(1,2, \ldots, n)$, such that

$$
C_{I V I F C S}\left(\widehat{A}_{\sigma(j-1)}, \widehat{B}_{\sigma(j-1)}\right) \geqslant C_{I V I F C S}\left(\widehat{A}_{\sigma(j)}, \widehat{B}_{\sigma(j)}\right), \quad j=2,3, \ldots, n,
$$

and $C_{I V I F C S}\left(\widehat{A}_{j}, \widehat{B}_{j}\right)$ denotes the interval-valued intuitionistic fuzzy cosine similarity measure for the element $x_{j}$.

The main advantages of the OWIVIFCS measure are that it is not only a straightforward generalization of measure defined in Eq. (18), but also it can relive (or intensify) the influence of unduly large or unduly small cosine similarity value on aggregation result by assigning them low (or high) weights as per our requirements.

Now consider the following numerical example to understand the computation procedure more clearly.

EXAMPLE 1. Let

$$
A=\left\{\begin{array}{c}
\left\langle x_{1},[0.5,0.6],[0.2,0.3]\right\rangle,\left\langle x_{2},[0.2,0.4],[0.4,0.5]\right\rangle \\
\left\langle x_{3},[0.4,0.6],[0.2,0.4]\right\rangle,\left\langle x_{4},[0.3,0.5],[0.2,0.4]\right\rangle \\
\left\langle x_{5},[0.5,0.6],[0.1,0.3]\right\rangle
\end{array}\right\}
$$

and

$$
B=\left\{\begin{array}{c}
\left\langle x_{1},[0.3,0.5],[0.4,0.5]\right\rangle,\left\langle x_{2},[0.5,0.6],[0.2,0.3]\right\rangle, \\
\left\langle x_{3},[0.3,0.4],[0.4,0.6]\right\rangle,\left\langle x_{4},[0.4,0.5],[0.1,0.2]\right\rangle, \\
\left\langle x_{5},[0.2,0.6],[0.3,0.4]\right\rangle
\end{array}\right\}
$$

be two interval-valued intuitionistic fuzzy sets. Further, assume that $U=(0.3,0.7)$ and $V=(0.5,0.5)$ are two weight vectors. The weighted reduced IFSs corresponding to IVIFSs $A$ and $B$ are obtained as

$$
\begin{aligned}
\widehat{A}= & \left\{\left\langle x_{1}, 0.57,0.25\right\rangle,\left\langle x_{2}, 0.34,0.45\right\rangle,\left\langle x_{3}, 0.54,0.30\right\rangle,\left\langle x_{4}, 0.44,0.30\right\rangle,\right. \\
& \left.\left\langle x_{5}, 0.57,0.20\right\rangle\right\}
\end{aligned}
$$

and

$$
\begin{aligned}
\widehat{B}= & \left\{\left\langle x_{1}, 0.44,0.45\right\rangle,\left\langle x_{2}, 0.57,0.25\right\rangle,\left\langle x_{3}, 0.37,0.50\right\rangle,\left\langle x_{4}, 0.47,0.15\right\rangle,\right. \\
& \left.\left\langle x_{5}, 0.48,0.35\right\rangle\right\}
\end{aligned}
$$

Then by Eq. (18), we get

$$
\begin{array}{ll}
C_{I V I F C S}\left(\widehat{A}_{1}, \widehat{B}_{1}\right)=0.9255, & C_{I V I F C S}\left(\widehat{A}_{2}, \widehat{B}_{2}\right)=0.8824 \\
C_{I V I F C S}\left(\widehat{A}_{3}, \widehat{B}_{3}\right)=0.9139, & C_{I V I F C S}\left(\widehat{A}_{4}, \widehat{B}_{4}\right)=0.9500 \\
C_{I V I F C S}\left(\widehat{A}_{5}, \widehat{B}_{5}\right)=0.9582 . &
\end{array}
$$


Table 1

Values of $C_{\text {OWIVIFCS }}^{\delta}(\widehat{A}, \widehat{B})$ for different values of $\delta$.

\begin{tabular}{cccccccccc}
\hline$\delta$ & 0.2 & 0.7 & 1 & 2 & 5 & 7 & 9 & 15 & 25 \\
\hline$C_{\text {OWIVIFCS }}^{\delta}$ & 0.9303 & 0.9304 & 0.9305 & 0.9309 & 0.9317 & 0.9325 & 0.9331 & 0.9349 & 0.9374 \\
\hline
\end{tabular}

Thus,

$$
\begin{array}{ll}
C_{I V I F C S}\left(\widehat{A}_{\sigma(1)}, \widehat{B}_{\sigma(1)}\right)=0.9582, & C_{I V I F C S}\left(\widehat{A}_{\sigma(2)}, \widehat{B}_{\sigma(2)}\right)=0.9500, \\
C_{I V I F C S}\left(\widehat{A}_{\sigma(3)}, \widehat{B}_{\sigma(3)}\right)=0.9255, & C_{I V I F C S}\left(\widehat{A}_{\sigma(4)}, \widehat{B}_{\sigma(4)}\right)=0.9139, \\
C_{I V I F C S}\left(\widehat{A}_{\sigma(5)}, \widehat{B}_{\sigma(5)}\right)=0.8824 . &
\end{array}
$$

Assume the weighting vector of ordered positions of cosine similarity measures $C_{I V I F C S}\left(\widehat{A}_{j}, \widehat{B}_{j}\right)(j=1,2, \ldots, 5)$ is $w=(0.30,0.15,0.10,0.25,0.20)$.

Taking different values of $\delta$ in Eq. (36), we can get the similarity measure between IVIFSs $A$ and $B$. The values of $C_{I V I F C S}^{\delta}(A, B)$ for different values of $\delta$ are shown in Table 2.

\subsection{Properties of the Ordered Weighted Interval-Valued Intuitionistic Fuzzy Cosine Similarity (OWIVIFCS), $C_{\text {OWIVIFCS }}^{\delta}(\widehat{A}, \widehat{B})$}

The OWIVIFCS measure is commutative, monotonic, bounded, idempotent, nonnegative, and reflexive. These properties can be proved with the following theorems:

Theorem 7 (Commutativity-GOWA aggregation). Let $\widehat{A}=\left\{\left\langle x_{i}, \eta_{\widehat{A}}^{i}, \psi_{\widehat{A}}^{i}\right\rangle \mid x_{i} \in X\right\}$ and $\widehat{B}=\left\{\left\langle x, \eta_{\widehat{B}}^{i}, \psi_{\widehat{B}}^{i}\right\rangle \mid x_{i} \in X\right\}$ denote the weighted reduced IFSs of the IVIFSs $A$ and B. If $\left(C_{\text {IVIFCS }}^{\prime}\left(\widehat{A}_{1}, \widehat{B}_{1}\right), C_{\text {IVIFCS }}^{\prime}\left(\widehat{A}_{2}, \widehat{B}_{2}\right), \ldots, C_{I V I F C S}^{\prime}\left(\widehat{A}_{n}, \widehat{B}_{n}\right)\right)$ is any permutation of $\left(C_{I V I F C S}\left(\widehat{A}_{1}, \widehat{B}_{1}\right), C_{I V I F C S}\left(\widehat{A}_{2}, \widehat{B}_{2}\right), \ldots, C_{I V I F C S}\left(\widehat{A}_{n}, \widehat{B}_{n}\right)\right)$, then

$$
C_{\text {OWIVIFCS }}^{\delta^{\prime}}(\widehat{A}, \widehat{B})=C_{\text {OWIVIFCS }}^{\delta}(\widehat{A}, \widehat{B}) .
$$

Theorem 8 (Commutativity-similarity measure). Let $\widehat{A}=\left\{\left\langle x_{i}, \eta_{\widehat{A}}^{i}, \psi_{\widehat{A}}^{i}\right\rangle \mid x_{i} \in X\right\}$ and $\widehat{B}=\left\{\left\langle x, \eta_{\widehat{B}}^{i}, \psi_{\widehat{B}}^{i}\right\rangle \mid x_{i} \in X\right\}$ denote the weighted reduced IFSs of the IVIFSs $A$ and $B$. Then

$$
C_{\text {OWIVIFCS }}^{\delta}(\widehat{A}, \widehat{B})=C_{\text {OWIVIFCS }}^{\delta}(\widehat{B}, \widehat{A})
$$

Theorem 9 (Monotonicity-similarity measure). Let $\widehat{A}=\left\{\left\langle x_{i}, \eta_{\widehat{A}}^{i}, \psi_{\widehat{A}}^{i}\right\rangle \mid x_{i} \in X\right\} \widehat{B}=$ $\left\{\left\langle x, \eta_{\widehat{B}}^{i}, \psi_{\widehat{B}}^{i}\right\rangle \mid x_{i} \in X\right\}$ and $\widehat{C}=\left\{\left\langle x, \eta_{\widehat{C}}^{i}, \psi_{\widehat{C}}^{i}\right\rangle \mid x_{i} \in X\right\}$ denote the weighted reduced IFSs of the IVIFSs $A, B$ and C. If $C_{\text {IVIFCS }}\left(\widehat{A}_{i}, \widehat{B}_{i}\right) \leqslant C_{\text {IVIFCS }}\left(\widehat{A}_{i}, \widehat{C}_{i}\right) \forall i$, then

$$
C_{\text {OWIVIFCS }}^{\delta}(\widehat{A}, \widehat{B}) \leqslant C_{\text {OWIVIFCS }}^{\delta}(\widehat{A}, \widehat{C}),
$$


where $C_{\text {IVIFCS }}\left(\widehat{A}_{i}, \widehat{B}_{i}\right)$ is the interval-valued intuitionistic fuzzy cosine similarity measure between $A_{i}$ and $B_{i}$, and $C_{I V I F C S}\left(\widehat{A}_{i}, \widehat{C}_{i}\right)$ is the interval-valued intuitionistic fuzzy cosine similarity measure between $A_{i}$ and $C_{i}$.

Theorem 10 (Monotonicity-parameter $\delta$ ). Let $\widehat{A}=\left\{\left\langle x_{i}, \eta_{\widehat{A}}^{i}, \psi_{\widehat{A}}^{i}\right\rangle \mid x_{i} \in X\right\}$ and $\widehat{B}=$ $\left\{\left\langle x, \eta_{\widehat{B}}^{i}, \psi_{\widehat{B}}^{i}\right\rangle \mid x_{i} \in X\right\}$ denote the weighted reduced IFSs of the IVIFSs $A$ and B. If $\delta_{1} \leqslant \delta_{2}$, then

$$
C_{\text {OWIVIFCS }}^{\delta_{1}}(\widehat{A}, \widehat{B}) \leqslant C_{\text {OWIVIFCS }}^{\delta_{2}}(\widehat{A}, \widehat{B}) .
$$

Theorem 11 (Idempotency). Let $\widehat{A}=\left\{\left\langle x_{i}, \eta_{\widehat{A}}^{i}, \psi_{\widehat{A}}^{i}\right\rangle \mid x_{i} \in X\right\}$ and $\widehat{B}=\left\{\left\langle x, \eta_{\widehat{B}}^{i}, \psi_{\widehat{B}}^{i}\right\rangle \mid x_{i} \in\right.$ $X\}$ denote the weighted reduced IFSs of the IVIFSs $A$ and B. If $C_{I V I F C S}\left(\widehat{A}_{i}, \widehat{B}_{i}\right)=\theta \forall i$, then

$$
C_{\text {OWIVIFCS }}^{\delta}(\widehat{A}, \widehat{B})=\theta
$$

Theorem 12 (Nonnegativity). Let $\widehat{A}=\left\{\left\langle x_{i}, \eta_{\widehat{A}}^{i}, \psi_{\widehat{A}}^{i}\right\rangle \mid x_{i} \in X\right\}$ and $\widehat{B}=\left\{\left\langle x, \eta_{\widehat{B}}^{i}, \psi_{\widehat{B}}^{i}\right\rangle \mid x_{i} \in\right.$ $X\}$ denote the weighted reduced IFSs of the IVIFSs $A$ and $B$. Then

$$
0 \leqslant C_{\text {OWIVIFCS }}^{\delta}(\widehat{A}, \widehat{B}) \leqslant 1 .
$$

Theorem 13 (Reflexivity). Let $\widehat{A}=\left\{\left\langle x_{i}, \eta_{\widehat{A}}^{i}, \psi_{\widehat{A}}^{i}\right\rangle \mid x_{i} \in X\right\}$ denote the weighted reduced IFS of the IVIFS A. Then

$$
C_{\text {OWIVIFCS }}^{\delta}(\widehat{A}, \widehat{A})=1
$$

Note that the proofs of these theorems are straightforward and thus omitted here.

\subsection{Families of OWIVIFCS Measure}

By using the different manifestation of the weighting vector $w$ and parameter $\delta$, we can obtain a wide range of particular types of OWIVIFCS measures. The selection of a weighting vector $w$ and parameter $\delta$ depends on the decision maker's attitude towards specific considered problems.

\subsubsection{Analysing the Parameter $\delta$}

When we consider different values of the parameter $\delta$ in $C_{\text {OWIVIFCS }}^{\delta}(\widehat{A}, \widehat{B})$, we will get different special cases of the cosine similarity measure defined in Eq. (36). Some notable particular cases of $C_{\text {OWIVIFCS }}^{\delta}(\widehat{A}, \widehat{B})$ are given by:

1. If $\delta=1$, then the OWIVIFCS measure gives the ordered weighted interval-valued intuitionistic fuzzy arithmetic cosine similarity (OWIVIFACS) measure:

$$
C_{\text {OWIVIFCS }}^{\delta}(\widehat{A}, \widehat{B})=\sum_{j=1}^{n} w_{j}\left(C_{I V I F C S}\left(\widehat{A}_{\sigma(j)}, \widehat{B}_{\sigma(j)}\right)\right),
$$


where $(\sigma(1), \sigma(2), \ldots, \sigma(n))$ is any permutation of $(1,2, \ldots, n)$ such that the condition given in Eq. (37) holds. Note that if $w=(1 / n, 1 / n, \ldots, 1 / n)$ in Eq. (45), then we get interval-valued intuitionistic fuzzy cosine similarity (IVIFCS) measure given in Eq. (18). The weighted interval-valued intuitionistic fuzzy cosine similarity (WIVIFCS) measure (32) is obtained if the ordered position of $C_{I V I F C S}\left(\widehat{A}_{j}, \widehat{B}_{j}\right)$ is the same as the ordered position of the $C_{I V I F C S}\left(\widehat{A}_{\sigma(j)}, \widehat{B}_{\sigma(j)}\right)$.

2. If $\delta=2$, then the OWIVIFCS measure becomes

$$
C_{\text {OWIVIFCS }}^{\delta}(\widehat{A}, \widehat{B})=\sqrt{\sum_{j=1}^{n} w_{j}\left(C_{I V I F C S}\left(\widehat{A}_{\sigma(j)}, \widehat{B}_{\sigma(j)}\right)\right)^{2}},
$$

where $(\sigma(1), \sigma(2), \ldots, \sigma(n))$ is any permutation of $(1,2, \ldots, n)$ such that the condition given in Eq. (37). We call it the ordered weighted quadratic interval-valued intuitionistic fuzzy cosine similarity (OWQIVIFCS) measure.

3. If $\delta=3$, then the OWIVIFCS measure gives

$$
C_{\text {OWIVIFCS }}^{\delta}(\widehat{A}, \widehat{B})=\left[\sum_{j=1}^{n} w_{j}\left(C_{I V I F C S}\left(\widehat{A}_{\sigma(j)}, \widehat{B}_{\sigma(j)}\right)\right)^{3}\right]^{1 / 3},
$$

where $(\sigma(1), \sigma(2), \ldots, \sigma(n))$ is any permutation of $(1,2, \ldots, n)$ such that the condition given in Eq. (37). We call it the ordered weighted cubic interval-valued intuitionistic fuzzy cosine similarity (OWCIVIFCS) measure.

4. If $\delta \rightarrow 0$, then the OWIVIFCS measure reduces

$$
C_{\text {OWIVIFCS }}^{\delta}(\widehat{A}, \widehat{B})=\prod_{j=1}^{n}\left(C_{I V I F C S}\left(\widehat{A}_{\sigma(j)}, \widehat{B}_{\sigma(j)}\right)\right)^{w_{j}}
$$

where $(\sigma(1), \sigma(2), \ldots, \sigma(n))$ is any permutation of $(1,2, \ldots, n)$ such that the condition given in Eq. (37) holds. We call it the ordered weighted interval-valued intuitionistic fuzzy geometric cosine similarity (OWIVIFGCS) measure. Note that the OWIVIFGCS measure can only be used in the situation when all the individual similarity measures are different from 0 , i.e. $C_{I V I F C S}\left(\widehat{A}_{j}, \widehat{B}_{j}\right) \neq 0 \forall j$.

\subsubsection{Analysing the Weighting Vector $w$}

By considering the different selections of the weighting vector, we are able to analyse the cosine similarity measure between two interval-valued intuitionistic fuzzy sets from min. similarity to max. similarity.

1. If $w_{1}=1$ and $w_{j}=0 \forall j \neq 1$, the $C_{\text {OWIVIFCS }}^{\delta}(\widehat{A}, \widehat{B})$ gives interval-valued intuitionistic fuzzy maximum cosine similarity (IVIFMAXCS) measure.

2. If $w_{n}=1$ and $w_{j}=0 \forall j \neq n$, the $C_{\text {OWIVIFCS }}^{\delta}(\widehat{A}, \widehat{B})$ gives interval-valued intuitionistic fuzzy minimum cosine similarity (IVIFMINCS) measure. 
3. More generally, if $w_{k}=1$ and $w_{j}=0 \forall j \neq k$, we obtain interval-valued intuitionistic fuzzy step cosine similarity (IVIFSTEPCS) measure.

4. If $w_{j}=1 / n \forall j$, then the $C_{O W I V I F C S}^{\delta}(\widehat{A}, \widehat{B})$ becomes interval-valued intuitionistic fuzzy normalized cosine similarity (IVIFNORCS) measure. Especially, if $\delta=1$, IVIFNORCS gives the IVIFCS measure defined in Eq. (18), and if $\delta \rightarrow 0$, we get the interval-valued intuitionistic fuzzy geometric cosine similarity (IVIFGCS) measure.

5. The WIVIFCS measure is obtained when the ordered position of the $i$ is the same as the ordered position of the $j$.

6. If $w_{(n+1) / 2}=1, w_{j}=0, j \neq(n+1) / 2, n$ is odd; or $w_{n / 2}=1, w_{j}=0, j \neq n / 2$, $n$ is even, then the $C_{\text {OWIVIFCS }}^{\delta}(\widehat{A}, \widehat{B})$ is reduced to interval-valued intuitionistic fuzzy median cosine similarity (IVIFMEDCS) measure.

7. If $w_{1}=w_{n}=0$ and $w_{j}=1 /(n-2) \forall j \neq 1, n$, then $C_{\text {OWIVIFCS }}^{\delta}(\widehat{A}, \widehat{B})$ reduces to interval-valued intuitionistic fuzzy Olympic cosine similarity (IVIFOLMCS) measure. Note that if $n=3$ or $n=4$, the IVIFOLMCS measure gives the interval-valued intuitionistic fuzzy median cosine similarity (IVIFMEDCS) measure.

8. If $w_{j}=0$ for $j=1,2, \ldots, k, n, n-1, \ldots, n-k+1$; and for all others, $w_{j}=1 /(n-$ $2 k)$, where $k<n / 2$, then the $C_{\text {OWIVIFCS }}^{\delta}(\widehat{A}, \widehat{B})$ gives the interval-valued intuitionistic fuzzy general Olympic cosine similarity (IVIFGOLYCS) measure.

9. If $w_{j}=1 / m$ for $k \leqslant j \leqslant k+m-1$, and $w_{j}=0 \forall j \geqslant k+m$ and $j<k$, then $C_{\text {OWIVIFCS }}^{\delta}(\widehat{A}, \widehat{B})$ gives to interval-valued intuitionistic fuzzy window cosine similarity (IVIFWINCS) measure.

It is interesting to note that the OWIVIFCS measure can be further generalized by using the quasi-OWA operator in place of GOWA. We call it quasi-OWIVIFCS measure. It can be defined as follows:

Definition 13 (Quasi-ordered weighted interval-valued intuitionistic fuzzy cosine similarity measure). A quasi-OWIVIFCS measure based on the weighted reduced IFSs of IVIFSs is a mapping Quasi-OWIVIFCS : IVIFS $(X) \times \operatorname{IVIFS}(X) \rightarrow[0,1]$ that has an associated weighting vector $w=\left(w_{1}, w_{2}, \ldots, w_{n}\right)$ with $w_{j} \in[0,1]$ and $\sum_{j=1}^{n} w_{j}=1$, and defined by

$$
\text { quasi-C } \operatorname{OWIVIFCS}(\widehat{A}, \widehat{B})=g^{-1}\left[\sum_{j=1}^{n} w_{j} g\left(C_{I V I F C S}\left(\widehat{A}_{\sigma(j)}, \widehat{B}_{\sigma(j)}\right)\right)\right]
$$

where $(\sigma(1), \sigma(2), \ldots, \sigma(n))$ is any permutation of $(1,2, \ldots, n)$, such that

$$
C_{I V I F C S}\left(\widehat{A}_{\sigma(j-1)}, \widehat{B}_{\sigma(j-1)}\right) \geqslant C_{I V I F C S}\left(\widehat{A}_{\sigma(j)}, \widehat{B}_{\sigma(j)}\right), \quad j=2,3, \ldots, n,
$$

and $C_{I V I F C S}\left(\widehat{A}_{j}, \widehat{B}_{j}\right)$ denotes the interval-valued intuitionistic fuzzy cosine similarity measure for element $x_{j}$ and $g$ is a strictly continuous monotonic function.

As we can see, when $g(t)=t^{\delta}$, then the quasi-C $C_{\text {OWIVIFCS }}(\widehat{A}, \widehat{B})$ measure becomes OWIVIFCS measure. Also, note that all the properties and particular cases associated with OWIVIFCS measure are also applicable in the quasi-OWIVIFCS measure. 
Further, by assigning different functions to $g(t)$, we can obtain a wide range of new cosine similarity measures for interval-valued intuitionistic fuzzy sets.

\section{For example:}

(1) when $g_{1}(t)=\sin ((\pi / 2) t), g_{2}(t)=\cos ((\pi / 2) t)$ and $g_{3}(t)=\tan ((\pi / 2) t)$, we obtain trigonometric-OWIVIFCS measures given by

$$
\begin{aligned}
& \sin -C_{\text {OWIVIFCS }}(\widehat{A}, \widehat{B})=\frac{2}{\pi} \arcsin \left[\sum_{j=1}^{n} w_{j} \sin \left(\frac{\pi}{2} C_{\text {IVIFCS }}\left(\widehat{A}_{\sigma(j)}, \widehat{B}_{\sigma(j)}\right)\right)\right] \\
& \cos -C_{\text {OWIVIFCS }}(\widehat{A}, \widehat{B})=\frac{2}{\pi} \arccos \left[\sum_{j=1}^{n} w_{j} \cos \left(\frac{\pi}{2} C_{\text {IVIFCS }}\left(\widehat{A}_{\sigma(j)}, \widehat{B}_{\sigma(j)}\right)\right)\right], \\
& \tan -C_{\text {OWIVIFCS }}(\widehat{A}, \widehat{B})=\frac{2}{\pi} \arctan \left[\sum_{j=1}^{n} w_{j} \tan \left(\frac{\pi}{2} C_{I V I F C S}\left(\widehat{A}_{\sigma(j)}, \widehat{B}_{\sigma(j)}\right)\right)\right] .
\end{aligned}
$$

(2) When $g(t)=\lambda^{t}, \lambda>0, \lambda \neq 1$, we get exponential-OWIVIFCS measure as

$$
\exp -C_{O W I V I F C S}(\widehat{A}, \widehat{B})=\log _{\lambda}\left[\sum_{j=1}^{n} w_{j} \lambda C_{I V I F C S}\left(\widehat{A}_{\sigma(j)}, \widehat{B}_{\sigma(j)}\right)\right] .
$$

(3) If $g(t)=\lambda^{1 / t}, \lambda>0, \lambda \neq 1$, then the quasi-OWIVIFCS measure gives a radicalOWIVIFCS measure

$$
\operatorname{Rad}-C_{O W I V I F C S}(\widehat{A}, \widehat{B})=\left[\log _{\lambda}\left(\sum_{j=1}^{n} w_{j} \lambda^{1 / C_{I V I F C S}\left(\widehat{A}_{\sigma(j)}, \widehat{B}_{\sigma(j)}\right)}\right)\right]^{-1}
$$

The OWIVIFCS measure can be applied to solve different problems, including decision-making, medical diagnosis, pattern recognition, engineering, and economics. In the next section, we present an application of the proposed OWIVIFCS measure to solve the multiple criteria decision-making problem with the interval-valued intuitionistic fuzzy information.

\section{Multiple Criteria Decision-Making Method Based on OWIVIFCS Measure}

IVIFS is a suitable tool for better modelling the imperfectly defined facts and data, as well as imprecise knowledge. In this section, we present a 5-step method to solve a multiple criteria decision-making problem under an interval-valued intuitionistic fuzzy environment. 


\subsection{Method}

Let $O=\left(O_{1}, O_{2}, \ldots, O_{m}\right)$ be a set of options and $C=\left(C_{1}, C_{2}, \ldots, C_{n}\right)$ be a set of criteria. Assume that the characteristics of the option $O_{k}$ in terms criteria $C$, entered by the decision-maker, are represented by the following IVIFSs:

$$
O_{k}=\left\{\left\langle C_{i},\left[\eta_{O_{k}}^{-}\left(C_{i}\right), \eta_{O_{k}}^{+}\left(C_{i}\right)\right],\left[\psi_{O_{k}}^{-}\left(C_{i}\right), \psi_{O_{k}}^{+}\left(C_{i}\right)\right]\right\rangle \mid C_{i} \in C\right\}, \quad k=1,2, \ldots, m,
$$

where $\left[\eta_{O_{k}}^{-}\left(C_{i}\right), \eta_{O_{k}}^{+}\left(C_{i}\right)\right]$ indicates the degree that the option $O_{k}$ satisfies the criterion $C_{i}$, and $\left[\psi_{O_{k}}^{-}\left(C_{i}\right), \psi_{O_{k}}^{+}\left(C_{i}\right)\right]$ denotes the degree that the option $O_{k}$ does not satisfy the criterion $C_{i}$.

Using the OWIVIFCS measure defined in Eq. (36), we set out the following approach to solve multiple criteria interval-valued intuitionistic fuzzy decision-making problems.

Step 1: Find the ideal solution $P$ defined as follows:

$$
P=\left\{\left\langle C_{i},\left[\eta_{P}^{-}\left(C_{i}\right), \eta_{P}^{+}\left(C_{i}\right)\right],\left[\psi_{P}^{-}\left(C_{i}\right), \psi_{P}^{+}\left(C_{i}\right)\right]\right\rangle \mid C_{i} \in C\right\}
$$

where for each $i=1,2, \ldots, n$,

$$
\begin{aligned}
& \left\langle\left[\eta_{P}^{-}\left(C_{i}\right), \eta_{P}^{+}\left(C_{i}\right)\right],\left[\psi_{P}^{-}\left(C_{i}\right), \psi_{P}^{+}\left(C_{i}\right)\right]\right\rangle \\
& \quad=\left\langle\left[\max _{k} \eta_{O_{k}}^{-}\left(C_{i}\right), \max _{k} \eta_{O_{k}}^{+}\left(C_{i}\right)\right],\left[\min _{k} \psi_{O_{k}}^{-}\left(C_{i}\right), \min _{k} \psi_{O_{k}}^{+}\left(C_{i}\right)\right]\right\rangle .
\end{aligned}
$$

Step 2: Calculate the interval-valued intuitionistic fuzzy cosine similarity measures $C_{I V I F C S}\left(\widehat{P}_{i}, \widehat{O}_{k i}\right)$ for each option $O_{k}(k=1,2, \ldots, m)$ by the following formula:

$$
\begin{aligned}
& C_{\text {IVIFCS }}\left(\widehat{P}_{i}, \widehat{O}_{k i}\right) \\
& \quad=\left[\frac{\eta_{\widehat{P}}^{i} \eta_{\widehat{O}_{k}}^{i}+\psi_{\widehat{P}}^{i} \psi_{\widehat{O}_{k}}^{i}+\left(1-\eta_{\widehat{P}}^{i}-\psi_{\widehat{P}}^{i}\right)\left(1-\eta_{\widehat{O}_{k}}^{i}-\psi_{\widehat{O}_{k}}^{i}\right)}{\sqrt{\left(\left(\eta_{\widehat{P}}^{i}\right)^{2}+\left(\psi_{\widehat{P}}^{i}\right)^{2}+\left(1-\eta_{\widehat{P}}^{i}-\psi_{\widehat{P}}^{i}\right)^{2}\right)} \sqrt{\left(\left(\eta_{\widehat{O}_{k}}^{i}\right)^{2}+\left(\psi_{\widehat{O}_{k}}^{i}\right)^{2}+\left(1-\eta_{\widehat{O}_{k}}^{i}-\psi_{\widehat{O}_{k}}^{i}\right)^{2}\right)}}\right],
\end{aligned}
$$

where

$$
\begin{aligned}
& \eta_{\widehat{O}_{k}}^{i}=u_{1} \eta_{O_{k}}^{-}\left(C_{i}\right)+u_{2} \eta_{O_{k}}^{+}\left(C_{i}\right), v_{\widehat{O}_{k}}^{i}=v_{1} \psi_{O_{k}}^{-}\left(C_{i}\right)+v_{2} \psi_{O_{k}}^{+}\left(C_{i}\right) ; \\
& \eta_{\widehat{P}}^{i}=u_{1} \eta_{P}^{-}\left(C_{i}\right)+u_{2} \eta_{P}^{+}\left(C_{i}\right), v_{\widehat{P}}^{i}=v_{1} \psi_{P}^{-}\left(C_{i}\right)+v_{2} \psi_{P}^{+}\left(C_{i}\right) ; \\
& u_{1}, u_{2}, v_{1}, v_{2} \in[0,1], \quad u_{1}+u_{2}=1 \quad \text { and } \quad v_{1}+v_{2}=1 .
\end{aligned}
$$

Step 3: Utilize the OWIVIFCS measure

$$
C_{\text {OWIVIFCS }}^{\delta}\left(\widehat{P}, \widehat{O}_{k}\right)=\left[\sum_{j=1}^{n} w_{j}\left(C_{I V I F C S}\left(\widehat{P}_{\sigma(j)},\left(\widehat{O}_{k \sigma(j)}\right)\right)\right)^{\delta}\right]^{1 / \delta},
$$


to aggregate the IVIFCS measures, $C_{I V I F C S}\left(\widehat{P}_{i}, \widehat{O}_{k i}\right)$, into a collective value $C_{\text {OWIVIFCS }}^{\delta}\left(\widehat{P}, \widehat{O}_{k}\right)$ of the alternative $O_{k}$, where

$$
C_{I V I F C S}\left(\widehat{P}_{\sigma(j-1)},\left(\widehat{O}_{k \sigma(j-1)}\right)\right) \geqslant C_{I V I F C S}\left(\widehat{P}_{\sigma(j)},\left(\widehat{O}_{k \sigma(j)}\right)\right), \quad k=1,2, \ldots, m .
$$

Step 4: Rank all the alternatives $O_{k}(k=1,2, \ldots, m)$ in accordance with the values of $C_{\text {OWIVIFCS }}^{\delta}\left(\widehat{P}, \widehat{O}_{k}\right)$ in descending order and select the best one.

\subsection{Numerical Example}

In the following, we are going to consider a real-life numerical example to demonstrate the applicability of the proposed method to multiple criteria decision making. To do so, we consider below a contractor selection decision-making problem for road development.

Example 2. Chile is a South American country occupying a long, narrow strip of land between the Andes to the east and the Pacific Ocean to the west. In Chile, tourism has become one of the main sources of income for the people, especially living in its most extreme areas. In 2018, a record of a total of 7 million international tourists visited Chile. The online guestbook Lonely Planet listed 'Chile' as its number one tourist destination to visit in the year 2018. Chile is typically divided into three geographic areas: (1) Continental Chile (2) Insular Chile and (3) Chilean Antarctic Territory. Chile, with its unique natural features, attracts more and more tourists every year. The main attractions for tourists are places of natural beauty situated in the extreme areas of the country including San Pedro de Atacama, Valley of the Moon, Chungará Lake, Parinacota, Pomerape, Portillo, Valle Nevado, Termas de Chillán, Conguillío National Park, Laguna San Rafael National Park, Valparaíso. In order to promote and stimulate growth within Chile's tourism sector, the Chilean government wants to develop many road-building projects either to preserve the roads which are already built or to undertake new roads. To do so, the Chilean government had issued the global tender in leading newspapers to select the contractor for these projects and considered the following six criteria for it: (1) financial status $\left(C_{1}\right)$; (2) organizational experience $\left(C_{2}\right)$; (3) past performance and knowledge $\left(C_{3}\right)$; (4) ability to deal with unanticipated problems $\left(C_{4}\right)$; (5) completion time $\left(C_{5}\right)$; and $(6)$ technical capability $\left(C_{6}\right)$. The five contractors (i.e. options), namely, (1) Sacyr Global Company $\left(O_{1}\right)$; (2) Eurovia $\left(\mathrm{O}_{2}\right)$; (3) Bechtel Group Inc. $\left(\mathrm{O}_{3}\right)$; (4) Acciona Construction $\left(\mathrm{O}_{4}\right)$; and (5) Ecoroads $\left(\mathrm{O}_{5}\right)$, bid for these projects. Here, the aim of the government is to recognize the best contractor among $O_{k},(k=1,2,3,4,5)$ who fulfills the desired goals of the project. A committee of experts (appointed by government officials) evaluates these contractors based on criteria $C_{i}$ and provides their evaluation information in the form of following interval-valued intuitionistic fuzzy sets:

$$
\begin{aligned}
O_{1}= & \left\{\left\langle\begin{array}{r}
C_{1},[0.2,0.3], \\
{[0.4,0.5]}
\end{array}\right\rangle,\left\langle\begin{array}{r}
C_{2},[0.6,0.7] \\
{[0.2,0.3]}
\end{array}\right\rangle,\left\langle\begin{array}{r}
C_{3},[0.4,0.5], \\
{[0.2,0.4]}
\end{array}\right\rangle,\left\langle\begin{array}{r}
C_{4},[0.7,0.8], \\
{[0.1,0.2]}
\end{array}\right),\right. \\
& \left.\left\langle\begin{array}{r}
C_{5},[0.1,0.3], \\
{[0.5,0.6]}
\end{array}\right\rangle,\left\langle\begin{array}{r}
C_{6},[0.5,0.7], \\
{[0.2,0.3]}
\end{array}\right\}\right\} ;
\end{aligned}
$$




$$
\begin{aligned}
& O_{2}=\left\{\left\langle\begin{array}{r}
C_{1},[0.6,0.7], \\
{[0.2,0.3]}
\end{array}\right\rangle,\left\langle\begin{array}{r}
C_{2},[0.5,0.6], \\
{[0.1,0.3]}
\end{array}\right\rangle,\left\langle\begin{array}{r}
C_{3},[0.6,0.7], \\
{[0.2,0.3]}
\end{array}\right\rangle,\left\langle\begin{array}{r}
C_{4},[0.6,0.7], \\
{[0.1,0.2]}
\end{array}\right\rangle,\right. \\
& \left.\left\langle\begin{array}{r}
C_{5},[0.3,0.4] \\
{[0.5,0.6]}
\end{array}\right),\left\langle\begin{array}{r}
C_{6},[0.4,0.7] \\
{[0.1,0.2]}
\end{array}\right)\right\} \\
& O_{3}=\left\{\left\langle\begin{array}{r}
C_{1},[0.4,0.5] \\
{[0.3,0.4]}
\end{array}\right\rangle,\left\langle\begin{array}{r}
C_{2},[0.7,0.8] \\
{[0.1,0.2]}
\end{array}\right),\left(\begin{array}{r}
C_{3},[0.5,0.6] \\
{[0.3,0.4]}
\end{array}\right),\left\langle\begin{array}{r}
C_{4},[0.6,0.7], \\
{[0.1,0.3]}
\end{array}\right\rangle\right. \text {, } \\
& \left.\left\langle\begin{array}{r}
C_{5},[0.4,0.5], \\
{[0.3,0.4]}
\end{array}\right),\left\langle\begin{array}{r}
C_{6},[0.3,0.5], \\
{[0.1,0.3]}
\end{array}\right)\right\} \text {; } \\
& O_{4}=\left\{\left\langle\begin{array}{r}
C_{1},[0.6,0.7], \\
{[0.2,0.3]}
\end{array}\right\rangle,\left\langle\begin{array}{r}
C_{2},[0.5,0.7], \\
{[0.1,0.3]}
\end{array}\right\rangle,\left\langle\begin{array}{r}
C_{3},[0.7,0.8], \\
{[0.1,0.2]}
\end{array}\right),\left\langle\begin{array}{r}
C_{4},[0.3,0.4], \\
{[0.1,0.2]}
\end{array}\right\rangle\right. \text {, } \\
& \left.\left\langle\begin{array}{r}
C_{5},[0.5,0.6], \\
{[0.1,0.3]}
\end{array}\right),\left\langle\begin{array}{r}
C_{6},[0.7,0.8], \\
{[0.1,0.2]}
\end{array}\right\rangle\right\} \\
& O_{5}=\left\{\left\langle\begin{array}{r}
C_{1},[0.5,0.6], \\
{[0.3,0.4]}
\end{array}\right\rangle,\left\langle\begin{array}{r}
C_{2},[0.3,0.4] \\
{[0.3,0.5]}
\end{array}\right\rangle,\left\langle\begin{array}{r}
C_{3},[0.6,0.7], \\
{[0.1,0.3]}
\end{array}\right\rangle,\left\langle\begin{array}{r}
C_{4},[0.6,0.8], \\
{[0.1,0.2]}
\end{array}\right\rangle\right. \text {, } \\
& \left.\left\langle\begin{array}{r}
C_{5},[0.6,0.7], \\
{[0.2,0.3]}
\end{array}\right),\left(\begin{array}{r}
C_{6},[0.5,0.6] \\
{[0.2,0.4]}
\end{array}\right)\right\} \text {. }
\end{aligned}
$$

The step-wise decision-making process as follows:

Step 1: We obtain the ideal solution $P$ as:

$$
P=\left\{\begin{array}{c}
\left\langle C_{1},[0.6,0.7],[0.2,0.3]\right\rangle,\left\langle C_{2},[0.7,0.8],[0.1,0.2]\right\rangle, \\
\left\langle C_{3},[0.7,0.8],[0.1,0.2]\right\rangle,\left\langle C_{4},[0.7,0.8],[0.1,0.2]\right\rangle, \\
\left\langle C_{5},[0.6,0.7],[0.1,0.3]\right\rangle,\left\langle C_{6},[0.7,0.8],[0.1,0.2]\right\rangle
\end{array}\right\} .
$$

Step 2: Using the formula defined in Eq. (58) to calculate $C_{I V I F C S}\left(\widehat{P}_{i}, \widehat{O}_{k i}\right)$ for each option $O_{k}(k=1,2, \ldots, 5)$, taking different values of $u_{1}, u_{2}, v_{1}$ and $v_{2}$. We have the following standard cases:

(i) Optimistic case: Let $u_{1}=0, u_{2}=1, v_{1}=1$ and $v_{2}=0$, we get Table 2 .

(ii) Pessimistic case: Let $u_{1}=1, u_{2}=0, v_{1}=0$ and $v_{2}=1$, we get Table 3 .

(iii) Neutral case: Let $u_{1}=0.5, u_{2}=0.5, v_{1}=0.5$ and $v_{2}=0.5$, Table 4 is obtained:

Step 3: We use the formula given in Eq. (59) to obtain the values of $C_{\text {OWIVIFCS }}^{\delta}\left(\widehat{P}, \widehat{O}_{k}\right)$ in all three cases, by taking $\delta=0.2, \delta=0.5, \delta=1, \delta=2, \delta=5, \delta=10$, respectively. Here, it is possible to consider different methods based on the OWIVIFCS measure for the selection of the contractor. In this example, we consider IVIFMAXCS, IVIFMINCS, IVIFNORCS, IVIFMEDCS, IVIFOLMCS, IVIFWINCS measures. We get the following Tables 5, 6, 7:

(i) Optimistic case (see Table 6). 
Table 2

Values of $C_{I V I F C S}\left(\widehat{P}_{i}, \widehat{O}_{k i}\right)$ for different options $O_{k}(k=1,2, \ldots, m)$.

\begin{tabular}{cccccc}
\hline$C_{I V I F C S}\left(\widehat{P}_{1}, \widehat{O}_{11}\right)$ & $C_{I V I F C S}\left(\widehat{P}_{2}, \widehat{O}_{12}\right)$ & $C_{I V I F C S}\left(\widehat{P}_{3}, \widehat{O}_{13}\right)$ & $C_{I V I F C S}\left(\widehat{P}_{4}, \widehat{O}_{14}\right)$ & $C_{I V I F C S}\left(\widehat{P}_{5}, \widehat{O}_{15}\right)$ & $C_{I V I F C S}\left(\widehat{P}_{6}, \widehat{O}_{16}\right)$ \\
0.7468 & 0.9883 & 0.8986 & 1.0000 & 0.6623 & 0.9883 \\
$C_{I V I F C S}\left(\widehat{P}_{1}, \widehat{O}_{21}\right)$ & $C_{I V I F C S}\left(\widehat{P}_{2}, \widehat{O}_{22}\right)$ & $C_{I V I F C S}\left(\widehat{P}_{3}, \widehat{O}_{23}\right)$ & $C_{I V I F C S}\left(\widehat{P}_{4}, \widehat{O}_{24}\right)$ & $C_{I V I F C S}\left(\widehat{P}_{5}, \widehat{O}_{25}\right)$ & $C_{I V I F C S}\left(\widehat{P}_{6}, \widehat{O}_{26}\right)$ \\
1.0000 & 0.9437 & 0.9883 & 0.9910 & 0.7349 & 0.9883 \\
$C_{I V I F C S}\left(\widehat{P}_{1}, \widehat{O}_{31}\right)$ & $C_{I V I F C S}\left(\widehat{P}_{2}, \widehat{O}_{32}\right)$ & $C_{I V I F C S}\left(\widehat{P}_{3}, \widehat{O}_{33}\right)$ & $C_{I V I F C S}\left(\widehat{P}_{4}, \widehat{O}_{34}\right)$ & $C_{I V I F C S}\left(\widehat{P}_{5}, \widehat{O}_{35}\right)$ & $C_{I V I F C S}\left(\widehat{P}_{6}, \widehat{O}_{36}\right)$ \\
0.9492 & 0.9437 & 0.9437 & 0.9883 & 0.9272 & 0.8547 \\
$C_{I V I F C S}\left(\widehat{P}_{1}, \widehat{O}_{41}\right)$ & $C_{I V I F C S}\left(\widehat{P}_{2}, \widehat{O}_{42}\right)$ & $C_{I V I F C S}\left(\widehat{P}_{3}, \widehat{O}_{43}\right)$ & $C_{I V I F C S}\left(\widehat{P}_{4}, \widehat{O}_{44}\right)$ & $C_{I V I F C S}\left(\widehat{P}_{5}, \widehat{O}_{45}\right)$ & $C_{I V I F C S}\left(\widehat{P}_{6}, \widehat{O}_{46}\right)$ \\
1.0000 & 0.9883 & 1.0000 & 0.7218 & 0.9832 & 1.0000 \\
$C_{I V I F C S}\left(\widehat{P}_{1}, \widehat{O}_{51}\right)$ & $C_{I V I F C S}\left(\widehat{P}_{2}, \widehat{O}_{52}\right)$ & $C_{I V I F C S}\left(\widehat{P}_{3}, \widehat{O}_{53}\right)$ & $C_{I V I F C S}\left(\widehat{P}_{4}, \widehat{O}_{54}\right)$ & $C_{I V I F C S}\left(\widehat{P}_{5}, \widehat{O}_{55}\right)$ & $C_{I V I F C S}\left(\widehat{P}_{6}, \widehat{O}_{56}\right)$ \\
0.9832 & 0.8022 & 0.9883 & 1.0000 & 0.9815 & 0.9650 \\
\hline
\end{tabular}

Table 3

Values of $C_{I V I F C S}\left(\widehat{P}_{i}, \widehat{O}_{k i}\right)$ for different options $O_{k}(k=1,2, \ldots, m)$.

\begin{tabular}{cccccc}
\hline$C_{I V I F C S}\left(\widehat{P}_{1}, \widehat{O}_{11}\right)$ & $C_{I V I F C S}\left(\widehat{P}_{2}, \widehat{O}_{12}\right)$ & $C_{I V I F C S}\left(\widehat{P}_{3}, \widehat{O}_{13}\right)$ & $C_{I V I F C S}\left(\widehat{P}_{4}, \widehat{O}_{14}\right)$ & $C_{I V I F C S}\left(\widehat{P}_{5}, \widehat{O}_{15}\right)$ & $C_{I V I F C S}\left(\widehat{P}_{6}, \widehat{O}_{16}\right)$ \\
0.7175 & 0.9832 & 0.8619 & 1.0000 & 0.5870 & 0.9492 \\
$C_{I V I F C S}\left(\widehat{P}_{1}, \widehat{O}_{21}\right)$ & $C_{I V I F C S}\left(\widehat{P}_{2}, \widehat{O}_{22}\right)$ & $C_{I V I F C S}\left(\widehat{P}_{3}, \widehat{O}_{23}\right)$ & $C_{I V I F C S}\left(\widehat{P}_{4}, \widehat{O}_{24}\right)$ & $C_{I V I F C S}\left(\widehat{P}_{5}, \widehat{O}_{25}\right)$ & $C_{I V I F C S}\left(\widehat{P}_{6}, \widehat{O}_{26}\right)$ \\
1.0000 & 0.9492 & 0.9832 & 0.9487 & 0.8043 & 0.8165 \\
$C_{I V I F C S}\left(\widehat{P}_{1}, \widehat{O}_{31}\right)$ & $C_{I V I F C S}\left(\widehat{P}_{2}, \widehat{O}_{32}\right)$ & $C_{I V I F C S}\left(\widehat{P}_{3}, \widehat{O}_{33}\right)$ & $C_{I V I F C S}\left(\widehat{P}_{4}, \widehat{O}_{34}\right)$ & $C_{I V I F C S}\left(\widehat{P}_{5}, \widehat{O}_{35}\right)$ & $C_{I V I F C S}\left(\widehat{P}_{6}, \widehat{O}_{36}\right)$ \\
0.9338 & 1.0000 & 0.9239 & 0.9832 & 0.9338 & 0.7235 \\
$C_{I V I F C S}\left(\widehat{P}_{1}, \widehat{O}_{41}\right)$ & $C_{I V I F C S}\left(\widehat{P}_{2}, \widehat{O}_{42}\right)$ & $C_{I V I F C S}\left(\widehat{P}_{3}, \widehat{O}_{43}\right)$ & $C_{I V I F C S}\left(\widehat{P}_{4}, \widehat{O}_{44}\right)$ & $C_{I V I F C S}\left(\widehat{P}_{5}, \widehat{O}_{45}\right)$ & $C_{I V I F C S}\left(\widehat{P}_{6}, \widehat{O}_{46}\right)$ \\
1.0000 & 0.9492 & 1.0000 & 0.6623 & 0.9806 & 1.0000 \\
$C_{I V I F C S}\left(\widehat{P}_{1}, \widehat{O}_{51}\right)$ & $C_{I V I F C S}\left(\widehat{P}_{2}, \widehat{O}_{52}\right)$ & $C_{I V I F C S}\left(\widehat{P}_{3}, \widehat{O}_{53}\right)$ & $C_{I V I F C S}\left(\widehat{P}_{4}, \widehat{O}_{54}\right)$ & $C_{I V I F C S}\left(\widehat{P}_{5}, \widehat{O}_{55}\right)$ & $C_{I V I F C S}\left(\widehat{P}_{6}, \widehat{O}_{56}\right)$ \\
0.9783 & 0.7285 & 0.9832 & 0.9847 & 1.0000 & 0.9239 \\
\hline
\end{tabular}

Table 4

Values of $C_{I V I F C S}\left(\widehat{P}_{i}, \widehat{O}_{k i}\right)$ for different options $O_{k}(k=1,2, \ldots, m)$.

\begin{tabular}{cccccc}
\hline$C_{I V I F C S}\left(\widehat{P}_{1}, \widehat{O}_{11}\right)$ & $C_{I V I F C S}\left(\widehat{P}_{2}, \widehat{O}_{12}\right)$ & $C_{I V I F C S}\left(\widehat{P}_{3}, \widehat{O}_{13}\right)$ & $C_{I V I F C S}\left(\widehat{P}_{4}, \widehat{O}_{14}\right)$ & $C_{I V I F C S}\left(\widehat{P}_{5}, \widehat{O}_{15}\right)$ & $C_{I V I F C S}\left(\widehat{P}_{6}, \widehat{O}_{16}\right)$ \\
0.7276 & 0.9858 & 0.8867 & 1.0000 & 0.6261 & 0.9766 \\
$C_{I V I F C S}\left(\widehat{P}_{1}, \widehat{O}_{21}\right)$ & $C_{I V I F C S}\left(\widehat{P}_{2}, \widehat{O}_{22}\right)$ & $C_{I V I F C S}\left(\widehat{P}_{3}, \widehat{O}_{23}\right)$ & $C_{I V I F C S}\left(\widehat{P}_{4}, \widehat{O}_{24}\right)$ & $C_{I V I F C S}\left(\widehat{P}_{5}, \widehat{O}_{25}\right)$ & $C_{I V I F C S}\left(\widehat{P}_{6}, \widehat{O}_{26}\right)$ \\
1.0000 & 0.9492 & 0.9858 & 0.9866 & 0.7674 & 0.9358 \\
$C_{I V I F C S}\left(\widehat{P}_{1}, \widehat{O}_{31}\right)$ & $C_{I V I F C S}\left(\widehat{P}_{2}, \widehat{O}_{32}\right)$ & $C_{I V I F C S}\left(\widehat{P}_{3}, \widehat{O}_{33}\right)$ & $C_{I V I F C S}\left(\widehat{P}_{4}, \widehat{O}_{34}\right)$ & $C_{I V I F C S}\left(\widehat{P}_{5}, \widehat{O}_{35}\right)$ & $C_{I V I F C S}\left(\widehat{P}_{6}, \widehat{O}_{36}\right)$ \\
0.9410 & 1.0000 & 0.9337 & 0.9913 & 0.9329 & 0.7995 \\
$C_{I V I F C S}\left(\widehat{P}_{1}, \widehat{O}_{41}\right)$ & $C_{I V I F C S}\left(\widehat{P}_{2}, \widehat{O}_{42}\right)$ & $C_{I V I F C S}\left(\widehat{P}_{3}, \widehat{O}_{43}\right)$ & $C_{I V I F C S}\left(\widehat{P}_{4}, \widehat{O}_{44}\right)$ & $C_{I V I F C S}\left(\widehat{P}_{5}, \widehat{O}_{45}\right)$ & $C_{I V I F C S}\left(\widehat{P}_{6}, \widehat{O}_{46}\right)$ \\
1.0000 & 0.9772 & 1.0000 & 0.6910 & 0.9815 & 1.0000 \\
$C_{I V I F C S}\left(\widehat{P}_{1}, \widehat{O}_{51}\right)$ & $C_{I V I F C S}\left(\widehat{P}_{2}, \widehat{O}_{52}\right)$ & $C_{I V I F C S}\left(\widehat{P}_{3}, \widehat{O}_{53}\right)$ & $C_{I V I F C S}\left(\widehat{P}_{4}, \widehat{O}_{54}\right)$ & $C_{I V I F C S}\left(\widehat{P}_{5}, \widehat{O}_{55}\right)$ & $C_{I V I F C S}\left(\widehat{P}_{6}, \widehat{O}_{56}\right)$ \\
0.9805 & 0.7670 & 0.9913 & 0.9970 & 0.9949 & 0.9509 \\
\hline
\end{tabular}

(ii) Pessimistic case (see Table 7).

(iii) Neutral case (see Table 8).

Step 4: Rank all the options $O_{k}(k=1,2, \ldots, m)$ in accordance with the values of $C_{\text {OWIVIFCS }}^{\delta}\left(\widehat{P}, \widehat{O}_{k}\right)$ in descending order. The results are presented in Table 8.

As we can see, depending on the cosine similarity measure used, the ranking order of the available options is different. Therefore, depending on the similarity measure employed, the results may lead to different decisions. In this problem, the IVIFMAXCS is the most optimistic cosine similarity measure because it considers only the highest similarity value. On the other hand, IVIFMINCS is the most pessimistic one. The IVIFNORCS is a neutral measure because it gives the same weights to all the characteristics. 
Table 5

Values of $C_{\text {OWIVIFCS }}^{\delta}\left(\widehat{P}, \widehat{O}_{k}\right)$ under different similarity measures.

\begin{tabular}{|c|c|c|c|c|c|c|}
\hline & IVIFMA & IVIFMI & IVIFNC & IVIFME & IVIFOI & IVIFWINCS \\
\hline$\delta=0.2$ & $C_{\text {OWIVIFCS }}^{\delta}\left(\widehat{P}, \widehat{O}_{1}\right) 1.0000$ & 0.6623 & 0.8723 & 0.9883 & 0.9010 & 0.9010 \\
\hline & $C_{\text {OWIVIFCS }}^{\delta}\left(\widehat{P}, \widehat{O}_{2}\right) 1.0000$ & 0.7349 & 0.9364 & 0.9883 & 0.9770 & 0.9770 \\
\hline & $C_{\text {OWIVIFCS }}^{\delta}\left(\widehat{P}, \widehat{O}_{3}\right) 1.0000$ & 0.8547 & 0.9430 & 0.9492 & 0.9521 & 0.9521 \\
\hline & $C_{\text {OWIVIFCS }}^{\delta}\left(\widehat{P}, \widehat{O}_{4}\right) 1.0000$ & 0.7218 & 0.9439 & 1.0000 & 0.9929 & 0.9929 \\
\hline & $C_{\text {OWIVIFCS }}^{\delta}\left(\widehat{P}, \widehat{O}_{5}\right) 1.0000$ & 0.8022 & 0.9512 & 0.9832 & 0.9795 & 0.9795 \\
\hline & $C_{\text {OWIVIFCS }}^{\delta}\left(\widehat{P}, \widehat{O}_{1}\right) 1.0000$ & 0.6623 & 0.8755 & 0.9883 & 0.9027 & 0.9027 \\
\hline$\delta=0.5$ & $C_{\text {OWIVIFCS }}^{\delta}\left(\widehat{P}, \widehat{O}_{2}\right) 1.0000$ & 0.7349 & 0.9380 & 0.9883 & 0.9771 & 0.9771 \\
\hline & $C_{\text {OWIVIFCS }}^{\delta}\left(\widehat{P}, \widehat{O}_{3}\right) 1.0000$ & 0.8547 & 0.9434 & 0.9492 & 0.9522 & 0.9522 \\
\hline & $C_{\text {OWIVIFCS }}^{\delta}\left(\widehat{P}, \widehat{O}_{4}\right) 1.0000$ & 0.7218 & 0.9458 & 1.0000 & 0.9929 & 0.9929 \\
\hline & $C_{\text {OWIVIFCS }}^{\delta}\left(\widehat{P}, \widehat{O}_{5}\right) 1.0000$ & 0.8022 & 0.9521 & 0.9832 & 0.9795 & 0.9795 \\
\hline & $C_{O W I V I F C S}^{\delta}\left(\widehat{P}, \widehat{O}_{1}\right) 1.0000$ & 0.6623 & 0.8807 & 0.9883 & 0.9055 & 0.9055 \\
\hline$\delta=1$ & $C_{\text {OWIVIFCS }}^{\delta}\left(\widehat{P}, \widehat{O}_{2}\right) 1.0000$ & 0.7349 & 0.9406 & 0.9883 & 0.9771 & 0.9771 \\
\hline & $C_{\text {OWIVIFCS }}^{\delta}\left(\widehat{P}, \widehat{O}_{3}\right) 1.0000$ & 0.8547 & 0.9440 & 0.9492 & 0.9524 & 0.9524 \\
\hline & $C_{\text {OWIVIFCS }}^{\delta}\left(\widehat{P}, \widehat{O}_{4}\right) 1.0000$ & 0.7218 & 0.9489 & 1.0000 & 0.9929 & 0.9929 \\
\hline & $C_{\text {OWIVIFCS }}^{\delta}\left(\widehat{P}, \widehat{O}_{5}\right) 1.0000$ & 0.8022 & 0.9534 & 0.9832 & 0.9795 & 0.9795 \\
\hline & $C_{\text {OWIVIFCS }}^{\delta}\left(\widehat{P}, \widehat{O}_{1}\right) 1.0000$ & 0.6623 & 0.8904 & 0.9883 & 0.9109 & 0.9109 \\
\hline$\delta=2$ & $C_{\text {OWIVIFCS }}^{\delta}\left(\widehat{P}, \widehat{O}_{2}\right) 1.0000$ & 0.7349 & 0.9452 & 0.9883 & 0.9773 & 0.9773 \\
\hline & $C_{\text {OWIVIFCS }}^{\delta}\left(\widehat{P}, \widehat{O}_{3}\right) 1.0000$ & 0.8547 & 0.9452 & 0.9492 & 0.9526 & 0.9526 \\
\hline & $C_{\text {OWIVIFCS }}^{\delta}\left(\widehat{P}, \widehat{O}_{4}\right) 1.0000$ & 0.7218 & 0.9543 & 1.0000 & 0.9929 & 0.9929 \\
\hline & $C_{\text {OWIVIFCS }}^{\delta}\left(\widehat{P}, \widehat{O}_{5}\right) 1.0000$ & 0.8022 & 0.9558 & 0.9832 & 0.9795 & 0.9795 \\
\hline & $C_{\text {OWIVIFCS }}^{\delta}\left(\widehat{P}, \widehat{O}_{1}\right) 1.0000$ & 0.6623 & 0.9142 & 0.9883 & 0.9247 & 0.9247 \\
\hline$\delta=5$ & $C_{\text {OWIVIFCS }}^{\delta}\left(\widehat{P}, \widehat{O}_{2}\right) 1.0000$ & 0.7349 & 0.9560 & 0.9883 & 0.9779 & 0.9779 \\
\hline & $C_{\text {OWIVIFCS }}^{\delta}\left(\widehat{P}, \widehat{O}_{3}\right) 1.0000$ & 0.8547 & 0.9486 & 0.9492 & 0.9535 & 0.9535 \\
\hline & $C_{\text {OWIVIFCS }}^{\delta}\left(\widehat{P}, \widehat{O}_{4}\right) 1.0000$ & 0.7218 & 0.9664 & 1.0000 & 0.9930 & 0.9930 \\
\hline & $C_{\text {OWIVIFCS }}^{\delta}\left(\widehat{P}, \widehat{O}_{5}\right) 1.0000$ & 0.8022 & 0.9619 & 0.9832 & 0.9797 & 0.9797 \\
\hline & $C_{\text {OWIVIFCS }}^{\delta}\left(\widehat{P}, \widehat{O}_{1}\right) 1.0000$ & 0.6623 & 0.9388 & 0.9883 & 0.9409 & 0.9409 \\
\hline$\delta=10$ & $C_{\text {OWIVIFCS }}^{\delta}\left(\widehat{P}, \widehat{O}_{2}\right) 1.0000$ & 0.7349 & 0.9666 & 0.9883 & 0.9788 & 0.9788 \\
\hline & $C_{\text {OWIVIFCS }}^{\delta}\left(\widehat{P}, \widehat{O}_{3}\right) 1.0000$ & 0.8547 & 0.9536 & 0.9492 & 0.9549 & 0.9549 \\
\hline & $C_{\text {OWIVIFCS }}^{\delta}\left(\widehat{P}, \widehat{O}_{4}\right) 1.0000$ & 0.7218 & 0.9774 & 1.0000 & 0.9931 & 0.9931 \\
\hline & $C_{\text {OWIVIFCS }}^{\delta}\left(\widehat{P}, \widehat{O}_{5}\right) 1.0000$ & 0.8022 & 0.9689 & 0.9832 & 0.9798 & 0.9798 \\
\hline
\end{tabular}

From Table 8, it is also interesting to note that the ranking orders may vary according to the attitude of the decision-makers towards the considered problem. It is a natural phenomenon in real-world decision-making problems. Because an optimistic decisionmaker always chooses the upper values of the membership intervals and lower values of the non-membership intervals in the measuring process, whereas a pessimistic decisionmaker considers the lower value of the membership interval and the upper value of the non-membership interval. A neutral decision-maker always concentrates on the central values of both intervals. 
Table 6

Values of $C_{O W I V I F C S}^{\delta}\left(\widehat{P}, \widehat{O}_{k}\right)$ under different similarity measures.

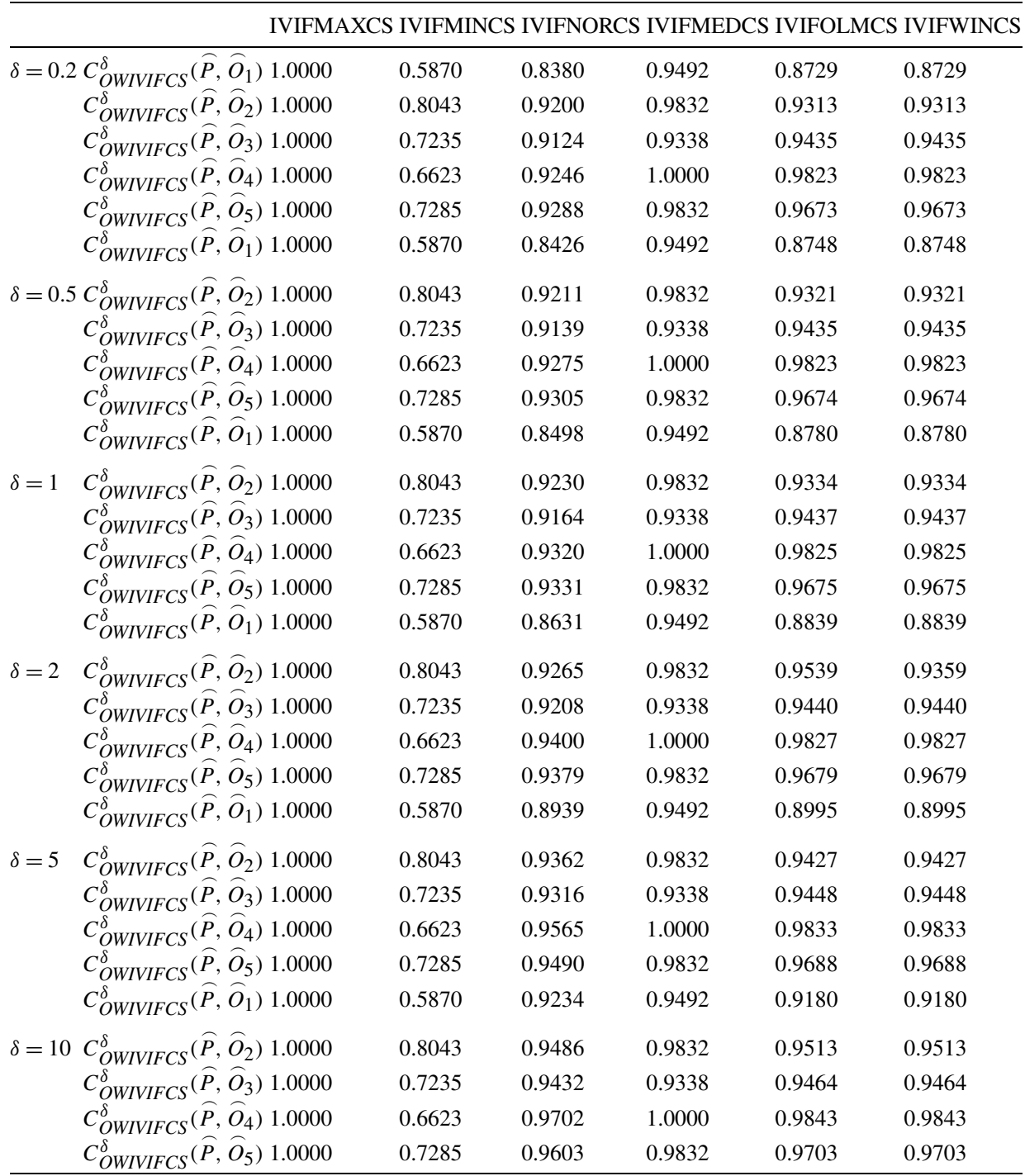

Further, in order to validate the performance of the developed different cosine similarity measures, a comparative study has been conducted and analysed in detail. Based on the normal distribution method (Xu, 2005), we obtain the optimal ordered weight vector $w=(0.1400,0.1710,0.1890,0.1890,0.1710,0.1400)$ associated with the criteria. Then, the similarity values and the corresponding ranking order of the options are summarized in Table 9.

From Table 9, it has been observed that the option $O_{5}$ or option $O_{4}$ is the best alternative in most cases, whereas the option $O_{1}$ is the worst one. It is also worth to note 
Table 7

Values of $C_{O W I V I F C S}^{\delta}\left(\widehat{P}, \widehat{O}_{k}\right)$ under different similarity measures.

\begin{tabular}{|c|c|c|c|c|c|c|}
\hline & IVIFMA & IVIFMI & IVIFNO & IVIFMI & IVIFOI & IVIFWINCS \\
\hline$\delta=0.2$ & $C_{\text {OWIVIFCS }}^{\delta}\left(\widehat{P}, \widehat{O}_{1}\right) 1.0000$ & 0.6261 & 0.8575 & 0.9766 & 0.8898 & 0.8898 \\
\hline & $C_{\text {OWIVIFCS }}^{\delta}\left(\widehat{P}, \widehat{O}_{2}\right) 1.0000$ & 0.7674 & 0.9346 & 0.9866 & 0.9641 & 0.9641 \\
\hline & $C_{\text {OWIVIFCS }}^{\delta}\left(\widehat{P}, \widehat{O}_{3}\right) 1.0000$ & 0.7995 & 0.9311 & 0.9410 & 0.9495 & 0.9495 \\
\hline & $C_{\text {OWIVIFCS }}^{\delta}\left(\widehat{P}, \widehat{O}_{4}\right) 1.0000$ & 0.6910 & 0.9454 & 1.0000 & 0.9896 & 0.9896 \\
\hline & $C_{\text {OWIVIFCS }}^{\delta}\left(\widehat{P}, \widehat{O}_{5}\right) 0.9970$ & 0.7670 & 0.9438 & 0.9913 & 0.9793 & 0.9793 \\
\hline & $C_{\text {OWIVIFCS }}^{\delta}\left(\widehat{P}, \widehat{O}_{1}\right) 1.0000$ & 0.6261 & 0.8614 & 0.9766 & 0.8918 & 0.8918 \\
\hline$\delta=0.5$ & $C_{\text {OWIVIFCS }}^{\delta}\left(\widehat{P}, \widehat{O}_{2}\right) 1.0000$ & 0.7674 & 0.9357 & 0.9866 & 0.9642 & 0.9642 \\
\hline & $C_{\text {OWIVIFCS }}^{\delta}\left(\widehat{P}, \widehat{O}_{3}\right) 1.0000$ & 0.7995 & 0.9319 & 0.9410 & 0.9496 & 0.9496 \\
\hline & $C_{\text {OWIVIFCS }}^{\delta}\left(\widehat{P}, \widehat{O}_{4}\right) 1.0000$ & 0.6910 & 0.9378 & 1.0000 & 0.9896 & 0.9896 \\
\hline & $C_{\text {OWIVIFCS }}^{\delta}\left(\widehat{P}, \widehat{O}_{5}\right) 0.9970$ & 0.7670 & 0.9450 & 0.9913 & 0.9793 & 0.9793 \\
\hline & $C_{O W I V I F C S}^{\delta}\left(\widehat{P}, \widehat{O}_{1}\right) 1.0000$ & 0.6261 & 0.8676 & 0.9766 & 0.8949 & 0.8949 \\
\hline$\delta=1$ & $C_{\text {OWIVIFCS }}^{\delta}\left(\widehat{P}, \widehat{O}_{2}\right) 1.0000$ & 0.7674 & 0.9375 & 0.9866 & 0.9643 & 0.9643 \\
\hline & $C_{\text {OWIVIFCS }}^{\delta}\left(\widehat{P}, \widehat{O}_{3}\right) 1.0000$ & 0.7995 & 0.9331 & 0.9410 & 0.9497 & 0.9497 \\
\hline & $C_{\text {OWIVIFCS }}^{\delta}\left(\widehat{P}, \widehat{O}_{4}\right) 1.0000$ & 0.6910 & 0.9416 & 1.0000 & 0.9897 & 0.9897 \\
\hline & $C_{\text {OWIVIFCS }}^{\delta}\left(\widehat{P}, \widehat{O}_{5}\right) 0.9970$ & 0.7670 & 0.9469 & 0.9913 & 0.9794 & 0.9794 \\
\hline & $C_{\text {OWIVIFCS }}^{\delta}\left(\widehat{P}, \widehat{O}_{1}\right) 1.0000$ & 0.6261 & 0.8787 & 0.9766 & 0.9009 & 0.9009 \\
\hline$\delta=2$ & $C_{\text {OWIVIFCS }}^{\delta}\left(\widehat{P}, \widehat{O}_{2}\right) 1.0000$ & 0.7674 & 0.9408 & 0.9866 & 0.9646 & 0.9646 \\
\hline & $C_{\text {OWIVIFCS }}^{\delta}\left(\widehat{P}, \widehat{O}_{3}\right) 1.0000$ & 0.7995 & 0.9354 & 0.9410 & 0.9500 & 0.9500 \\
\hline & $C_{\text {OWIVIFCS }}^{\delta}\left(\widehat{P}, \widehat{O}_{4}\right) 1.0000$ & 0.6910 & 0.9483 & 1.0000 & 0.9897 & 0.9897 \\
\hline & $C_{\text {OWIVIFCS }}^{\delta}\left(\widehat{P}, \widehat{O}_{5}\right) 0.9970$ & 0.7670 & 0.9505 & 0.9913 & 0.9796 & 0.9796 \\
\hline & $C_{\text {OWIVIFCS }}^{\delta}\left(\widehat{P}, \widehat{O}_{1}\right) 1.0000$ & 0.6261 & 0.9067 & 0.9766 & 0.9161 & 0.9161 \\
\hline$\delta=5$ & $C_{\text {OWIVIFCS }}^{\delta}\left(\widehat{P}, \widehat{O}_{2}\right) 1.0000$ & 0.7674 & 0.9491 & 0.9866 & 0.9654 & 0.9654 \\
\hline & $C_{\text {OWIVIFCS }}^{\delta}\left(\widehat{P}, \widehat{O}_{3}\right) 1.0000$ & 0.7995 & 0.9451 & 0.9410 & 0.9510 & 0.9510 \\
\hline & $C_{\text {OWIVIFCS }}^{\delta}\left(\widehat{P}, \widehat{O}_{4}\right) 1.0000$ & 0.6910 & 0.9626 & 1.0000 & 0.9899 & 0.9899 \\
\hline & $C_{\text {OWIVIFCS }}^{\delta}\left(\widehat{P}, \widehat{O}_{5}\right) 0.9970$ & 0.7670 & 0.9590 & 0.9913 & 0.9800 & 0.9800 \\
\hline & $C_{\text {OWIVIFCS }}^{\delta}\left(\widehat{P}, \widehat{O}_{1}\right) 1.0000$ & 0.6261 & 0.9336 & 0.9766 & 0.9334 & 0.9334 \\
\hline$\delta=10$ & $C_{\text {OWIVIFCS }}^{\delta}\left(\widehat{P}, \widehat{O}_{2}\right) 1.0000$ & 0.7674 & 0.9583 & 0.9866 & 0.9666 & 0.9666 \\
\hline & $C_{\text {OWIVIFCS }}^{\delta}\left(\widehat{P}, \widehat{O}_{3}\right) 1.0000$ & 0.7995 & 0.9494 & 0.9410 & 0.9527 & 0.9527 \\
\hline & $C_{\text {OWIVIFCS }}^{\delta}\left(\widehat{P}, \widehat{O}_{4}\right) 1.0000$ & 0.6910 & 0.9748 & 1.0000 & 0.9902 & 0.9902 \\
\hline & $C_{\text {OWIVIFCS }}^{\delta}\left(\widehat{P}, \widehat{O}_{5}\right) 0.9970$ & 0.7670 & 0.9680 & 0.9913 & 0.9807 & 0.9807 \\
\hline
\end{tabular}

that when $\tan -C_{\text {OWIVIFCS }}$ is used to calculate the aggregated similarity value for different options, then the obtained ranking order coincides with the ranking order attained by IVIFMAXCS in all three cases.

\section{Conclusions}

In this paper, we have suggested a new and flexible method for measuring the similarity between interval-valued intuitionistic fuzzy sets. Using the idea of weighted reduced in- 
Table 8

Ranking of options based on different similarity measures.

Optimistic case

\begin{tabular}{|c|c|c|c|c|c|}
\hline \multirow[t]{6}{*}{$\delta=0.2$} & IVIFMAXCS & $O_{1}=O_{2}=O_{3}=O_{4}=O_{5}$ & $\delta=0.5$ & IVIFMAXCS & $O_{1}=O_{2}=O_{3}=O_{4}=O_{5}$ \\
\hline & IVIFMINCS & $O_{3} \succ O_{5} \succ O_{2} \succ O_{4} \succ O_{1}$ & & IVIFMINCS & $O_{3} \succ O_{5} \succ O_{2} \succ O_{4} \succ O_{1}$ \\
\hline & IVIFNORCS & $O_{5} \succ O_{4} \succ O_{3} \succ O_{2} \succ O_{1}$ & & IVIFNORCS & $O_{5} \succ O_{4} \succ O_{3} \succ O_{2} \succ O_{1}$ \\
\hline & IVIFMEDCS & $O_{4} \succ O_{2}=O_{1} \succ O_{5} \succ O_{3}$ & & IVIFMEDCS & $O_{4} \succ O_{2}=O_{1} \succ O_{5} \succ O_{3}$ \\
\hline & IVIFOLMCS & $O_{4} \succ O_{5} \succ O_{2} \succ O_{3} \succ O_{1}$ & & IVIFOLMCS & $O_{4} \succ O_{5} \succ O_{2} \succ O_{3} \succ O_{1}$ \\
\hline & IVIFWINCS & $O_{4} \succ O_{5} \succ O_{2} \succ O_{3} \succ O_{1}$ & & IVIFWINCS & $O_{4} \succ O_{5} \succ O_{2} \succ O_{3} \succ O_{1}$ \\
\hline \multirow[t]{6}{*}{$\delta=1$} & IVIFMAXCS & $O_{1}=O_{2}=O_{3}=O_{4}=O_{5}$ & $\delta=2$ & IVIFMAXCS & $O_{1}=O_{2}=O_{3}=O_{4}=O_{5}$ \\
\hline & IVIFMINCS & $O_{3} \succ O_{5} \succ O_{2} \succ O_{4} \succ O_{1}$ & & IVIFMINCS & $O_{3} \succ O_{5} \succ O_{2} \succ O_{4} \succ O_{1}$ \\
\hline & IVIFNORCS & $O_{5} \succ O_{4} \succ O_{3} \succ O_{2} \succ O_{1}$ & & IVIFNORCS & $O_{5} \succ O_{4} \succ O_{3}=O_{2} \succ O_{1}$ \\
\hline & IVIFMEDCS & $O_{4} \succ O_{2}=O_{1} \succ O_{5} \succ O_{3}$ & & IVIFMEDCS & $O_{4} \succ O_{2}=O_{1} \succ O_{5} \succ O_{3}$ \\
\hline & IVIFOLMCS & $O_{4} \succ O_{5} \succ O_{2} \succ O_{3} \succ O_{1}$ & & IVIFOLMCS & $O_{4} \succ O_{5} \succ O_{2} \succ O_{3} \succ O_{1}$ \\
\hline & IVIFWINCS & $O_{4} \succ O_{2} \succ O_{5} \succ O_{1} \succ O_{3}$ & & IVIFWINCS & $O_{4} \succ O_{5} \succ O_{2} \succ O_{3} \succ O_{1}$ \\
\hline \multirow[t]{6}{*}{$\delta=5$} & IVIFMAXCS & $O_{1}=O_{2}=O_{3}=O_{4}=O_{5}$ & $\delta=10$ & IVIFMAXCS & $O_{1}=O_{2}=O_{3}=O_{4}=O_{5}$ \\
\hline & IVIFMINCS & $O_{3} \succ O_{5} \succ O_{2} \succ O_{4} \succ O_{1}$ & & IVIFMINCS & $O_{3} \succ O_{5} \succ O_{2} \succ O_{4} \succ O_{1}$ \\
\hline & IVIFNORCS & $O_{4} \succ O_{5} \succ O_{2} \succ O_{3} \succ O_{1}$ & & IVIFNORCS & $O_{4} \succ O_{5} \succ O_{2} \succ O_{3} \succ O_{1}$ \\
\hline & IVIFMEDCS & $O_{4} \succ O_{2}=O_{1} \succ O_{5} \succ O_{3}$ & & IVIFMEDCS & $O_{4} \succ O_{2}=O_{1} \succ O_{5} \succ O_{3}$ \\
\hline & IVIFOLMCS & $O_{4} \succ O_{5} \succ O_{2} \succ O_{3} \succ O_{1}$ & & IVIFOLMCS & $O_{4} \succ O_{5} \succ O_{2} \succ O_{3} \succ O_{1}$ \\
\hline & IVIFWINCS & $O_{4} \succ O_{5} \succ O_{2} \succ O_{3} \succ O_{1}$ & & IVIFWINCS & $O_{4} \succ O_{5} \succ O_{2} \succ O_{3} \succ O_{1}$ \\
\hline
\end{tabular}

Pessimistic case

\begin{tabular}{|c|c|c|c|c|c|}
\hline \multirow[t]{6}{*}{$\delta=0.2$} & IVIFMAXCS & $O_{1}=O_{2}=O_{3}=O_{4}=O_{5}$ & $\delta=0.5$ & IVIFMAXCS & $O_{1}=O_{2}=O_{3}=O_{4}=O_{5}$ \\
\hline & IVIFMINCS & $O_{2} \succ O_{5} \succ O_{3} \succ O_{4} \succ O_{1}$ & & IVIFMINCS & $O_{2} \succ O_{5} \succ O_{3} \succ O_{4} \succ O_{1}$ \\
\hline & IVIFNORCS & $O_{5} \succ O_{4} \succ O_{2} \succ O_{3} \succ O_{1}$ & & IVIFNORCS & $O_{5} \succ O_{4} \succ O_{2} \succ O_{3} \succ O_{1}$ \\
\hline & IVIFMEDCS & $O_{4} \succ O_{5}=O_{2} \succ O_{1} \succ O_{3}$ & & IVIFMEDCS & $O_{4} \succ O_{5}=O_{2} \succ O_{1} \succ O_{3}$ \\
\hline & IVIFOLMCS & $O_{4} \succ O_{5} \succ O_{3} \succ O_{2} \succ O_{1}$ & & IVIFOLMCS & $O_{4} \succ O_{5} \succ O_{3} \succ O_{2} \succ O_{1}$ \\
\hline & IVIFWINCS & $O_{4} \succ O_{5} \succ O_{3} \succ O_{2} \succ O_{1}$ & & IVIFWINCS & $O_{4} \succ O_{5} \succ O_{3} \succ O_{2} \succ O_{1}$ \\
\hline \multirow[t]{6}{*}{$\delta=1$} & IVIFMAXCS & $O_{1}=O_{2}=O_{3}=O_{4}=O_{5}$ & $\delta=2$ & IVIFMAXCS & $O_{1}=O_{2}=O_{3}=O_{4}=O_{5}$ \\
\hline & IVIFMINCS & $O_{2} \succ O_{5} \succ O_{3} \succ O_{4} \succ O_{1}$ & & IVIFMINCS & $O_{2} \succ O_{5} \succ O_{3} \succ O_{4} \succ O_{1}$ \\
\hline & IVIFNORCS & $O_{5} \succ O_{4} \succ O_{2} \succ O_{3} \succ O_{1}$ & & IVIFNORCS & $O_{4} \succ O_{5} \succ O_{2} \succ O_{3} \succ O_{1}$ \\
\hline & IVIFMEDCS & $O_{4} \succ O_{5}=O_{2} \succ O_{1} \succ O_{3}$ & & IVIFMEDCS & $O_{4} \succ O_{5}=O_{2} \succ O_{1} \succ O_{3}$ \\
\hline & IVIFOLMCS & $O_{4} \succ O_{5} \succ O_{3} \succ O_{2} \succ O_{1}$ & & IVIFOLMCS & $O_{4} \succ O_{5} \succ O_{2} \succ O_{3} \succ O_{1}$ \\
\hline & IVIFWINCS & $O_{4} \succ O_{5} \succ O_{3} \succ O_{2} \succ O_{1}$ & & IVIFWINCS & $O_{4} \succ O_{5} \succ O_{3} \succ O_{2} \succ O_{1}$ \\
\hline \multirow[t]{6}{*}{$\delta=5$} & IVIFMAXCS & $O_{1}=O_{2}=O_{3}=O_{4}=O_{5}$ & $\delta=10$ & IVIFMAXCS & $O_{1}=O_{2}=O_{3}=O_{4}=O_{5}$ \\
\hline & IVIFMINCS & $O_{2} \succ O_{5} \succ O_{3} \succ O_{4} \succ O_{1}$ & & IVIFMINCS & $O_{2} \succ O_{5} \succ O_{3} \succ O_{4} \succ O_{1}$ \\
\hline & IVIFNORCS & $O_{5} \succ O_{4} \succ O_{2} \succ O_{3} \succ O_{1}$ & & IVIFNORCS & $O_{4} \succ O_{2} \succ O_{2} \succ O_{3} \succ O_{1}$ \\
\hline & IVIFMEDCS & $O_{4} \succ O_{5}=O_{2} \succ O_{1} \succ O_{3}$ & & IVIFMEDCS & $O_{4} \succ O_{5}=O_{2} \succ O_{1} \succ O_{3}$ \\
\hline & IVIFOLMCS & $O_{4} \succ O_{5} \succ O_{3} \succ O_{2} \succ O_{1}$ & & IVIFOLMCS & $O_{4} \succ O_{5} \succ O_{2} \succ O_{3} \succ O_{1}$ \\
\hline & IVIFWINCS & $O_{4} \succ O_{5} \succ O_{3} \succ O_{2} \succ O_{1}$ & & IVIFWINCS & $O_{4} \succ O_{5} \succ O_{2} \succ O_{3} \succ O_{1}$ \\
\hline
\end{tabular}


Table 8

(continued)

\begin{tabular}{|c|c|c|c|c|c|}
\hline \multicolumn{6}{|c|}{ Neutral case } \\
\hline \multirow[t]{6}{*}{$\delta=0.2$} & IVIFMAXCS & $O_{1}=O_{2}=O_{3}=O_{4} \succ O_{5}$ & $\delta=0.5$ & IVIFMAXCS & $O_{1}=O_{2}=O_{3}=O_{4} \succ O_{5}$ \\
\hline & IVIFMINCS & $O_{3} \succ O_{2} \succ O_{5} \succ O_{4} \succ O_{1}$ & & IVIFMINCS & $O_{3} \succ O_{2} \succ O_{5} \succ O_{4} \succ O_{1}$ \\
\hline & IVIFNORCS & $O_{4} \succ O_{5} \succ O_{2} \succ O_{3} \succ O_{1}$ & & IVIFNORCS & $O_{5} \succ O_{4} \succ O_{2} \succ O_{3} \succ O_{1}$ \\
\hline & IVIFMEDCS & $O_{4} \succ O_{5} \succ O_{2} \succ O_{1} \succ O_{3}$ & & IVIFMEDCS & $O_{4} \succ O_{5} \succ O_{2} \succ O_{1} \succ O_{3}$ \\
\hline & IVIFOLMCS & $O_{4} \succ O_{5} \succ O_{2} \succ O_{3} \succ O_{1}$ & & IVIFOLMCS & $O_{4} \succ O_{5} \succ O_{2} \succ O_{3} \succ O_{1}$ \\
\hline & IVIFWINCS & $O_{4} \succ O_{5} \succ O_{2} \succ O_{3} \succ O_{1}$ & & IVIFWINCS & $O_{4} \succ O_{5} \succ O_{2} \succ O_{3} \succ O_{1}$ \\
\hline \multirow[t]{6}{*}{$\delta=1$} & IVIFMAXCS & $O_{1}=O_{2}=O_{3}=O_{4} \succ O_{5}$ & $\delta=2$ & IVIFMAXCS & $O_{1}=O_{2}=O_{3}=O_{4} \succ O_{5}$ \\
\hline & IVIFMINCS & $O_{3} \succ O_{2} \succ O_{5} \succ O_{4} \succ O_{1}$ & & IVIFMINCS & $O_{3} \succ O_{2} \succ O_{5} \succ O_{4} \succ O_{1}$ \\
\hline & IVIFNORCS & $O_{5} \succ O_{4} \succ O_{2} \succ O_{3} \succ O_{1}$ & & IVIFNORCS & $O_{5} \succ O_{4} \succ O_{2} \succ O_{3} \succ O_{1}$ \\
\hline & IVIFMEDCS & $O_{4} \succ O_{5} \succ O_{2} \succ O_{1} \succ O_{3}$ & & IVIFMEDCS & $O_{4} \succ O_{5} \succ O_{2} \succ O_{1} \succ O_{3}$ \\
\hline & IVIFOLMCS & $O_{4} \succ O_{5} \succ O_{2} \succ O_{3} \succ O_{1}$ & & IVIFOLMCS & $O_{4} \succ O_{5} \succ O_{2} \succ O_{3} \succ O_{1}$ \\
\hline & IVIFWINCS & $O_{4} \succ O_{5} \succ O_{2} \succ O_{3} \succ O_{1}$ & & IVIFWINCS & $O_{4} \succ O_{5} \succ O_{2} \succ O_{3} \succ O_{1}$ \\
\hline \multirow[t]{6}{*}{$\delta=5$} & IVIFMAXCS & $O_{1}=O_{2}=O_{3}=O_{4} \succ O_{5}$ & $\delta=10$ & IVIFMAXCS & $O_{1}=O_{2}=O_{3}=O_{4} \succ O_{5}$ \\
\hline & IVIFMINCS & $O_{3} \succ O_{2} \succ O_{5} \succ O_{4} \succ O_{1}$ & & IVIFMINCS & $O_{3} \succ O_{2} \succ O_{5} \succ O_{4} \succ O_{1}$ \\
\hline & IVIFNORCS & $O_{5} \succ O_{4} \succ O_{2} \succ O_{3} \succ O_{1}$ & & IVIFNORCS & $O_{5} \succ O_{4} \succ O_{2} \succ O_{3} \succ O_{1}$ \\
\hline & IVIFMEDCS & $O_{4} \succ O_{5} \succ O_{2} \succ O_{1} \succ O_{3}$ & & IVIFMEDCS & $O_{4} \succ O_{5} \succ O_{2} \succ O_{1} \succ O_{3}$ \\
\hline & IVIFOLMCS & $O_{4} \succ O_{5} \succ O_{2} \succ O_{3} \succ O_{1}$ & & IVIFOLMCS & $O_{4} \succ O_{5} \succ O_{2} \succ O_{3} \succ O_{1}$ \\
\hline & IVIFWINCS & $O_{4} \succ O_{5} \succ O_{2} \succ O_{3} \succ O_{1}$ & & IVIFWINCS & $O_{4} \succ O_{5} \succ O_{2} \succ O_{3} \succ O_{1}$ \\
\hline
\end{tabular}

tuitionistic fuzzy sets, the work has developed a new interval-valued intuitionistic fuzzy cosine similarity measure and proved some of its basic and essential properties. Its fundamental advantage is the ability to combine the subjective knowledge and attitudinal character of the decision-maker in measuring the process of similarity degree. Further, we have defined the ordered weighted interval-valued intuitionistic fuzzy cosine similarity measure. It is a similarity measure that uses the notion of GOWA in the normalization process of interval-valued intuitionistic fuzzy cosine similarity based on reduced intuitionistic fuzzy sets. This approach alleviates the influence of unduly large (or small) similarity values on aggregation results by assigning them low (or high) weights. Moreover, it also provides a parameterized family of cosine similarity measures from minimum cosine similarity to maximum cosine similarity between two interval-valued intuitionistic fuzzy sets. We have studied some of its main properties and particular cases.

The use of quasi-arithmetic means under this framework has also been studied to obtain the quasi-ordered weighted interval-valued intuitionistic fuzzy cosine similarity measure. This cosine similarity measure includes a wide range of particular cases, including the OWIVIFCS measure, the trigonometric-OWIVIFCS measures, the exponentialOWIVIFCS measure, and the radical-OWIVIFCS measure.

The newly developed interval-valued intuitionistic cosine similarity measures can be applied in different real-world decision problems. This paper has focused on multiple criteria decision-making problems. We have developed a decision-making method based on OWIVIFCS to solve real-world decision problems with interval-valued intuitionistic fuzzy information. Finally, a numerical example has been provided to illustrate the multiple criteria decision-making process. We have seen that this approach provides more information for decision making because it can consider a wide range of situations depending on 
Table 9

Ranking of options based on different cosine similarity measures under IVIF environment.

\begin{tabular}{|c|c|c|c|c|c|c|c|}
\hline & & $O_{1}$ & $\mathrm{O}_{2}$ & $\mathrm{O}_{3}$ & $\mathrm{O}_{4}$ & $\mathrm{O}_{5}$ & Ranking order \\
\hline \multirow[t]{10}{*}{ Optimistic } & OWIVIFACS & 0.8860 & 0.9468 & 0.9451 & 0.9560 & 0.9577 & $O_{5} \succ O_{4} \succ O_{2} \succ O_{3} \succ O_{1}$ \\
\hline & OWQIVIFCS & 0.8950 & 0.9509 & 0.9462 & 0.9606 & 0.9598 & $O_{4} \succ O_{5} \succ O_{2} \succ O_{3} \succ O_{1}$ \\
\hline & OWCIVIFCS & 0.9031 & 0.9544 & 0.9472 & 0.9646 & 0.9617 & $O_{4} \succ O_{5} \succ O_{2} \succ O_{3} \succ O_{1}$ \\
\hline & OWIVIFGCS & 0.8763 & 0.9422 & 0.9441 & 0.9505 & 0.9553 & $O_{5} \succ O_{4} \succ O_{3} \succ O_{2} \succ O_{1}$ \\
\hline & $\sin -C_{\text {OWIVIFCS }}$ & 0.8310 & 0.8983 & 0.9296 & 0.8963 & 0.9239 & $O_{3} \succ O_{5} \succ O_{2} \succ O_{4} \succ O_{1}$ \\
\hline & $\cos -C_{\text {OWIVIFCS }}$ & 0.8889 & 0.9479 & 0.9453 & 0.9572 & 0.9581 & $O_{5} \succ O_{4} \succ O_{3} \succ O_{2} \succ O_{1}$ \\
\hline & $\tan -C_{\text {OWIVIFCS }}$ & 1.0000 & 1.0000 & 1.0000 & 1.0000 & 1.0000 & $O_{1}=O_{2}=O_{3}=O_{4}=O_{5}$ \\
\hline & $\exp -C_{\text {OWIVIFCS }}$ & 0.8915 & 0.9494 & 0.9458 & 0.9589 & 0.9590 & $O_{5} \succ O_{4} \succ O_{2} \succ O_{3} \succ O_{1}$ \\
\hline & $\mathrm{Rad}-C_{\text {OWIVIFCS }}$ & 0.8566 & 0.9324 & 0.9421 & 0.9385 & 0.9507 & $O_{5} \succ O_{3} \succ O_{4} \succ O_{2} \succ O_{1}$ \\
\hline & OWIVIFCS & 0.8553 & 0.9258 & 0.9204 & 0.9404 & 0.9391 & $O_{4} \succ O_{5} \succ O_{2} \succ O_{3} \succ O_{1}$ \\
\hline \multirow[t]{9}{*}{ Pessimistic } & OWQIVIFCS & 0.8673 & 0.9292 & 0.9242 & 0.9472 & 0.9432 & $O_{4} \succ O_{5} \succ O_{2} \succ O_{3} \succ O_{1}$ \\
\hline & OWQIVIFCS & 0.8779 & 0.9323 & 0.9276 & 0.9529 & 0.9468 & $O_{4} \succ O_{5} \succ O_{2} \succ O_{3} \succ O_{1}$ \\
\hline & OWIVIFGCS & 0.8418 & 0.9223 & 0.9161 & 0.9321 & 0.9344 & $O_{5} \succ O_{4} \succ O_{2} \succ O_{3} \succ O_{1}$ \\
\hline & $\sin -C_{\text {OWIVIFCS }}$ & 0.7976 & 0.8921 & 0.8848 & 0.8729 & 0.8934 & $O_{5} \succ O_{2} \succ O_{3} \succ O_{4} \succ O_{1}$ \\
\hline & $\cos -C_{\text {OWIVIFCS }}$ & 0.8599 & 0.9265 & 0.9215 & 0.9425 & 0.9402 & $O_{4} \succ O_{5} \succ O_{2}>O_{3} \succ O_{1}$ \\
\hline & $\tan -C_{\text {OWIVIFCS }}$ & 1.0000 & 1.0000 & 1.0000 & 1.0000 & 1.0000 & $O_{1}=O_{2}=O_{3}=O_{4}=O_{5}$ \\
\hline & $\exp -C_{\text {OWIVIFCS }}$ & 0.8623 & 0.9280 & 0.9228 & 0.9446 & 0.9417 & $O_{4} \succ O_{5} \succ O_{2} \succ O_{3} \succ O_{1}$ \\
\hline & $\mathrm{Rad}-C_{\text {OWIVIFCS }}$ & 0.8128 & 0.9158 & 0.9071 & 0.9128 & 0.9244 & $O_{5} \succ O_{2} \succ O_{4} \succ O_{3} \succ O_{1}$ \\
\hline & OWIVIFCS & 0.8734 & 0.9419 & 0.9353 & 0.9493 & 0.9524 & $O_{5}>O_{4}>O_{2}>O_{3}>O_{1}$ \\
\hline \multirow[t]{8}{*}{ Neutral } & OWQIVIFCS & 0.8840 & 0.9448 & 0.9373 & 0.9551 & 0.9554 & $O_{5} \succ O_{4} \succ O_{2} \succ O_{3} \succ O_{1}$ \\
\hline & OWQIVIFCS & 0.8934 & 0.9474 & 0.9391 & 0.9599 & 0.9581 & $O_{4} \succ O_{5} \succ O_{2}>O_{3} \succ O_{1}$ \\
\hline & OWIVIFGCS & 0.8616 & 0.9387 & 0.9332 & 0.9425 & 0.9489 & $O_{5} \succ O_{4} \succ O_{2} \succ O_{3} \succ O_{1}$ \\
\hline & $\sin -C_{\text {OWIVIFCS }}$ & 0.8151 & 0.9064 & 0.9113 & 0.8847 & 0.9104 & $O_{5} \succ O_{4} \succ O_{3} \succ O_{2} \succ O_{1}$ \\
\hline & $\cos -C_{\text {OWIVIFCS }}$ & 0.8771 & 0.9426 & 0.9357 & 0.9510 & 0.9531 & $O_{5} \succ O_{4} \succ O_{2} \succ O_{3} \succ O_{1}$ \\
\hline & $\tan -C_{\text {OWIVIFCS }}$ & 1.0000 & 1.0000 & 1.0000 & 1.0000 & 0.9914 & $O_{1}=O_{2}=O_{3}=O_{4} \succ O_{5}$ \\
\hline & $\exp -C_{O W I V I F C S}$ & 0.8797 & 0.9437 & 0.9366 & 0.9530 & 0.9543 & $O_{5} \succ O_{4} \succ O_{2} \succ O_{3} \succ O_{1}$ \\
\hline & $\mathrm{Rad}-C_{\text {OWIVIFCS }}$ & 0.8372 & 0.9322 & 0.9291 & 0.9269 & 0.9419 & $O_{5} \succ O_{2} \succ O_{3} \succ O_{4} \succ O_{1}$ \\
\hline
\end{tabular}

the interest of decision-makers. The proposed approach also has some limitations. The developed interval-valued intuitionistic fuzzy cosine similarity measures can be utilized in situations where the degrees of membership and non-membership values take interval numerical values. However, in many real-life situations, linguistic variables are used to represent qualitative information. These similarity measures cannot be utilized under the linguistic environment. So, we need a further study of these similarity measures with linguistic interval-valued intuitionistic fuzzy information.

In future research, we expect to develop further extensions by using more complex formulations, including the use of inducing variables, probabilities, moving averages, power averages, Bonferroni means, etc. Other important issues to consider are consensus (Chiclana et al., 2013; del Moral et al., 2018), large-scale decision-making (Dong et al., 2018; Zhang et al., 2018), social networks decision making (Ureña et al., 2019). As we know, consensus measures play a very vital role in group decision-making problems. A high level of consensus among experts is needed before reaching a solution. We will also focus on the development of different consensus measures by utilizing proposed cosine similarity measures and study their applications in large-scale decision-making, social networks decision-making problems under different uncertain environments. 
Acknowledgements. We thank the anonymous reviewers for their insightful and constructive comments and suggestions that have led to an improved version of this paper.

\section{Funding}

The first author would like to acknowledge the Postdoctoral Research Financial support from Project 3170556 provided by the Chilean Government (Conicyt) through the Fondecyt Postdoctoral Program.

\section{References}

Aggarwal, A., Khan, I. (2016). On solving Atanassov's I-fuzzy linear programming problems: some variants of Angelov's model. Opsearch, 53(2), 375-389.

Atanassov, K.T. (1986). Intuitionistic fuzzy sets. Fuzzy Sets and Systems, 20(1), 87-96.

Atanassov, K.T. (1994). Operators over interval-valued intuitionistic fuzzy sets. Fuzzy Sets and Systems, 64(2), $159-174$.

Atanassov, K.T., Gargov, G. (1989). Interval-valued intuitionistic fuzzy sets. Fuzzy Sets and Systems, 31(3), 343-349.

Atanassov, K.T., Pasi, G., Yager, R.R. (2005). Intuitionistic fuzzy interpretations of multi-criteria multi-person and multi-measurement tool decision making. International Journal of System Science, 36(14), 859-868.

Bhattacharya, A. (1946). On a measure of divergence of two multinomial populations. Sankhya: The Indian Journal of Statistics, 7(4), 401-406.

Chen, H.Y., Liu, C.L., Sheng, Z.H. (2004). Induced ordered weighted harmonic averaging (IOWHA) operator and its application to combination forecasting method. Chinese Journal of Management Science, 12(5), 3540 .

Chiclana, F., Tapia García, J.M., del Moral, M.J., Herrera-Viedma, E. (2013). A statistical comparative study of different similarity measures of consensus in group decision making. Information Sciences, 221, 110-123.

De, S.K., Biswas, R., Roy, A.R. (2001). An application of intuitionistic fuzzy sets in medical diagnosis. Fuzzy Sets and Systems, 117(2), 209-213.

Denfeng, L., Chuntian, C. (2002). New similarity measures of intuitionistic fuzzy sets and application to pattern recognition. Pattern Recognition Letters, 23(1-3), 221-225.

Del Moral, M.J., Chiclana, F., Tapia, J.M., Herrera-Viedma, E. (2018). A comparative study on consensus measures in group decision making. International Journal of Intelligent Systems, 33(8), 1624-1638.

Dong, Y., Zhao, S., Zhang, H., Chiclana, F., Herrera-Viedma, E. (2018). A self-management mechanism for non-cooperative behaviors in large-scale group consensus reaching processes. IEEE Transactions on Fuzzy Systems, 26(6), 3276-3288.

Fodor, J., Marichal, J.L., Roubens, M. (1995). Characterization of the ordered weighted averaging operators. IEEE Transactions on Fuzzy Systems, 3(2), 236-240.

Hong, D.H., Kim, C. (1995). A note on similarity measures between vague sets and between elements. Information Sciences, 115(1-4), 83-96.

Hung, W.L., Yang, M.S. (2004). Similarity measures of intuitionistic fuzzy sets based on Hausdorff distances. Pattern Recognition Letter, 25(14), 1603-1611.

Hung, K.C., Wang, P. (2012). A new intuitionistic fuzzy cosine similarity measures for medical pattern recognition. In: Proceedings of International Conference on Business and Information (BAI-2012), Sapporo, Japan, July 3-5, 2012, pp. 1252-1261.

Liang, Z., Shi, P. (2003). Similarity measures on intuitionistic fuzzy sets. Pattern Recognition Letters, 24(15), 2687-2693.

Liu, C., Peng, B. (2017). A method for group decision making based on interval-valued intuitionistic fuzzy geometric distance measures. Informatica, 28(3), 453-470.

Liu, D., Chen, X., Peng, D. (2017). Interval-valued intuitionistic fuzzy ordered weighted cosine similarity measure and its application in investment decision making. Complexity. https://doi.org/10.1155/2017/1891923. 
Merigó, J.M. (2012). Probabilities in the OWA operator. Expert Systems with Applications, 39(13), 1145611467.

Merigó, J.M., Gil-Lafuente, A.M. (2010). New decision-making techniques and their application in the selection of financial products. Information Sciences, 180(11), 2085-2094.

Merigó, J.M., Casanovas, M. (2011). Decision making with distance measures and induced aggregation operators. Computer and Industrial Engineering, 60(1), 66-76.

Merigó, J.M., Gil-Lafuente, A.M. (2011a). Fuzzy induced generalized aggregation operators and its application in multi-person decision making. Expert Systems with Applications, 38(8), 9761-9772.

Merigó, J.M., Gil-Lafuente, A.M. (2011b). Decision making in sport management based on OWA operator. Expert Systems with Applications, 38(8), 10408-10413.

Merigó, J.M., Yager, R.R. (2013). Generalized moving averages, distance measures and OWA operators. International Journal of Uncertainty, Fuzziness and Knowledge-Based Systems, 21(4), 533-559.

Mitchell, H.B. (2003). On the Dengfeng-Chuntian similarity measure and its application to pattern recognition. Pattern Recognition Letters, 24(16), 3101-3104.

Park, D.G., Kwun, Y.C., Park, J.H., Park, Y. (2009). Correlation coefficient of interval-valued intuitionistic fuzzy sets and its application to multiple attribute group decision-making problems. Mathematical and Computer Modelling, 50(9-10), 1279-1293.

Salton, G., McGill, M.J. (1983). Introduction to Modern Information Retrieval. McGraw-Hill Book Company, New York.

Singh, P. (2012). A new method on measure of similarity between interval-valued intuitionistic fuzzy sets for pattern recognition. Journal of Applied \& Computational Mathematics, 1(1), 1-5.

Su, W., Yang, Y., Zhang, C., Zeng, S. (2013). Intuitionistic fuzzy decision making with similarity measures and OWA operator. International Journal of Uncertainty, Fuzziness and Knowledge-Based Systems, 21(2), 245-262.

Szmidt, E., Kacprzyk, J. (2013). Geometric similarity measures for intuitionistic fuzzy sets. In: Proceedings of the 8th Conference of the European Society for Fuzzy Logic and Technology (EUSFLAT-13), pp. 840-847.

Ureña, R., Kou, G., Dong, Y., Chiclana, F., Herrera-Viedma, E. (2019). A review on trust propagation and opinion dynamics in social networks and group decision-making frameworks. Information Sciences, 478, 461-475.

Verma, R.K., Sharma, B.D. (2011). Intuitionistic fuzzy sets: some new results. Notes on intuitionistic fuzzy sets, 17(3), 1-10.

Verma, R.K., Sharma, B.D. (2012). On generalized intuitionistic fuzzy divergence (relative information) and their properties. Journal of Uncertain Systems, 6(4), 308-320.

Verma, R.K., Sharma, B.D. (2013a). A new measure of inaccuracy with its application to multi-criteria decision making under intuitionistic fuzzy environment. Journal of Intelligent and Fuzzy Systems, 27(4), 1811-1824.

Verma, R.K., Sharma, B.D. (2013b). Intuitionistic fuzzy Jensen-Rényi divergence: applications to multipleattribute decision making. Informatica, 37(4), 399-409.

Verma, R.K., Sharma, B.D. (2013c). Exponential entropy on intuitionistic fuzzy sets. Kybernetika, 49(1), 111127.

Verma, R., Merigó, J.M. (2019). Variance measures with ordered weighted aggregation operators. International Journal of Intelligent Systems, 34(6), 1184-1205.

Vlochos, I.K., Sergiadis, G.D. (2007). Intuitionistic fuzzy information: applications to pattern recognition. Pattern Recognition Letters, 28(2), 197-206.

Wei, C.P., Wang, P., Zhang, Y. (2011). Entropy, similarity measure of interval-valued intuitionistic fuzzy sets and their applications. Information Sciences, 181(19), 4273-4286.

$\mathrm{Xu}, \mathrm{Z}$. (2005). An overview of methods for determining OWA weights. International Journal of Intelligent Systems, 20(8), 843-865.

$\mathrm{Xu}, \mathrm{Z}$. (2007). On similarity measures of interval-valued intuitionistic fuzzy sets and their application to pattern recognition. Journal of Southeast University, 23(1), 139-143.

$\mathrm{Xu}, \mathrm{Z}$. (2008). An overview of distance and similarity measures of intuitionistic fuzzy sets. International Journal of Uncertainty, Fuzziness, and Knowledge-Based Systems, 16(4), 529-555.

$\mathrm{Xu}, \mathrm{Z}$. (2010). A method based on distance measure for interval-valued intuitionistic fuzzy group decision making. Information Sciences, 180(1), 181-190.

Xu, Z. (2012). Fuzzy ordered distance measures. Fuzzy Optimization and Decision Making, 11(1), $73-97$.

Xu, Z., Chen, J. (2008). Ordered weighted distance measure. Journal of Systems Science and Systems Engineering, 17(4), 432-445. 
Xu, Z., Da, Q.L. (2002). The ordered weighted geometric averaging operators. International Journal of Intelligent Systems, 17(7), 709-716.

Yager, R.R. (1988). On ordered weighted averaging aggregation operators in multicriteria decision making. IEEE Transactions on Systems, Man and Cybernetics, 18(1), 183-190.

Yager, R.R. (1996). On the inclusion of variance in decision making under uncertainty. International Journal of Uncertainty, Fuzziness and Knowledge-Based Systems, 4(5), 401-409.

Yager, R.R. (2002). Heavy OWA operator. Fuzzy Optimization and Decision Making, 1(4), 379-397.

Yager, R.R. (2004). Generalized OWA aggregation operators. Fuzzy Optimization and Decision Making, 3(1), 93-107.

Yager, R.R. (2006). Generalizing variance to allow the inclusion of decision attitude in decision making under uncertainty. International Journal of Approximate Reasoning, 42(3), 137-158.

Ye, J. (2011). Cosine similarity measures for intuitionistic fuzzy sets and their applications. Mathematical and Computer Modelling, 53(1-2), 91-97.

Ye, J. (2012). Multicriteria decision-making method using the dice similarity measure based on the reduced interval-valued intuitionistic fuzzy sets of interval-valued intuitionistic fuzzy sets. Applied Mathematical Modelling, 36(9), 4466-4472.

Ye, J. (2013). Interval-valued intuitionistic fuzzy cosine similarity measures for multiple attribute decisionmaking. International Journal of General Systems, 42(8), 883-891.

Yu, Y., Xu, A., Merigó, J.M., Wang, H. (2015). Hesitant fuzzy linguistic ordered weighted distance operators for group decision making. Journal of Applied Mathematics and Computing, 49(1-2), 285-308.

Zadeh, L.A. (1965). Fuzzy sets. Information and Control, 8(3), 338-353.

Zeng, S., Palacios-Marques, D., Zhu, F. (2016). A new model for interactive group decision making with intuitionistic fuzzy preference relations. Informatica, 27(4), 911-928.

Zeng, S., Merigó, J.M., Palacios-Marqués, D., Jin, H., Gu, F. (2017). Intuitionistic fuzzy induced ordered weighted averaging distance operator and its application to decision making. Journal of Intelligent and Fuzzy Systems, 32(1), 11-22.

Zhang, Q., Yao, H., Zhang, Z. (2011). Some similarity measures of interval-valued intuitionistic fuzzy sets and application to pattern recognition. Applied Mechanics and Materials, 44-47, 3888-3892.

Zhang, H., Dong, Y., Herrera-Viedma, E. (2018). Consensus building for the heterogeneous large-scale GDM with the individual concerns and satisfactions. IEEE Transactions on Fuzzy Systems, 26(2), 884-898.

Zhang, C., Chen, C., Streimikiene, D., Balezentis, T. (2019). Intuitionistic fuzzy MULTIMOORA approach for multi-criteria assessment of the energy storage technologies. Applied Soft Computing, 79, 410-423.

Zhao, N., Xu, Z. (2016). Entropy measures for interval-valued intuitionistic fuzzy information from a comparative perspective and their application to decision making. Informatica, 27(1), 203-228.

Zhou, L., Chen, H., Liu, J. (2013). Continuous ordered weighted distance measure and its application to multiple attribute group decision making. Group Decision and Negotiation, 22(4), 739-758.

Zhou, L., Tao, Z., Chen, H., Liu, J. (2014). Intuitionistic fuzzy ordered weighted cosine similarity measure. Group Decision and Negotiation, 23(4), 879-900. 
R. Verma received the MSc degree in mathematics from Chaudhary Charan Singh University University, Meerut (U.P.), India, in 2006, and the PhD degree in applied mathematics with a speciality in information theory and computational intelligence techniques from Jaypee Institute of Information Technology (Deemed University), Noida (U.P.), India, in 2014. He is currently a postdoctoral research fellow at the Department of Management Control and Information Systems, University of Chile, Santiago, Chile. He has authored over 45 research articles published in refereed international journals including the International Journal of Intelligent Systems, Kybernetika, Journal of Intelligent and Fuzzy Systems, International Journal of Uncertainty, Fuzziness and Knowledge-Based Systems, International Journal of Machine Learning and Cybernetics, Neural Computing and Applications, Informatica. He has also authored five book chapters. He is currently interested in information measures, aggregation operators, multiple attribute group decision making, computational intelligence techniques, and computing with words.

J. M.Merigó (PhD 2009) is a professor at the School of Information, Systems, and Modelling at the Faculty of Engineering and Information Technology at the University of Technology, Sydney (Australia). Before joining UTS, he was a full professor at the Department of Management Control and Information Systems at the University of Chile. Previously, he was a senior research fellow at the Manchester Business School, University of Manchester (UK) and an assistant professor at the Department of Business Administration at the University of Barcelona (Spain). He holds a masters and a PhD degree in business administration from the University of Barcelona. He also holds a bachelors degree of science and of social sciences in economics and a masters degree in European business administration and business law from Lund University (Sweden).

He has published more than 400 articles in journals, books and conference proceedings. $\mathrm{He}$ is on the editorial board of several journals. He has also been a guest editor for several international journals, member of the scientific committee of several conferences and reviewer in a wide range of international journals. Recently (2015-2018), Clarivate Analytics (previously Thomson \& Reuters) has distinguished him as a highly cited researcher in computer science. He is currently interested in decision making, aggregation operators, computational intelligence, bibliometrics and applications in business and economics. 